\title{
Tracking Alzheimer's Disease
}

\author{
Paul M. Thompson ${ }^{a}$, Kiralee M. Hayashi ${ }^{a}$, Rebecca A. Dutton ${ }^{a}$, Ming-Chang Chiang ${ }^{\mathrm{a}}$, Alex \\ D. Leow ${ }^{a, b}$, Elizabeth R. Sowell ${ }^{a}$, Greig de Zubicaray ${ }^{c}$, James T. Becker ${ }^{d, e, f}$, Oscar L. \\ Lopez $^{\mathrm{d}}$, Howard J. Aizenstein ${ }^{\mathrm{e}}$, and Arthur W. Toga ${ }^{\mathrm{a}}$ \\ aDepartment of Neurology, Laboratory of Neuro Imaging, UCLA School of Medicine, Los Angeles, \\ California, USA \\ bNeuropsychiatric Institute, UCLA School of Medicine, Los Angeles, California, USA \\ ${ }^{\circ}$ Centre for Magnetic Resonance, University of Queensland, Brisbane, Australia \\ dDepartment of Neurology, University of Pittsburgh, Pittsburgh, Pennsylvania, USA \\ eDepartment of Psychiatry, University of Pittsburgh, Pittsburgh, Pennsylvania, USA \\ fDepartment of Psychology, University of Pittsburgh, Pittsburgh, Pennsylvania, USA
}

\section{Abstract}

Population-based brain mapping provides great insight into the trajectory of aging and dementia, as well as brain changes that normally occur over the human life span. We describe three novel brain mapping techniques, cortical thickness mapping, tensor-based morphometry (TBM), and hippocampal surface modeling, which offer enormous power for measuring disease progression in drug trials, and shed light on the neuroscience of brain degeneration in Alzheimer's disease (AD) and mild cognitive impairment (MCI).We report the first time-lapse maps of cortical atrophy spreading dynamically in the living brain, based on averaging data from populations of subjects with Alzheimer's disease and normal subjects imaged longitudinally with MRI. These dynamic sequences show a rapidly advancing wave of cortical atrophy sweeping from limbic and temporal cortices into higher-order association and ultimately primary sensorimotor areas, in a pattern that correlates with cognitive decline. A complementary technique, TBM, reveals the 3D profile of atrophic rates, at each point in the brain. A third technique, hippocampal surface modeling, plots the profile of shape alterations across the hippocampal surface. The three techniques provide moderate to highly automated analyses of images, have been validated on hundreds of scans, and are sensitive to clinically relevant changes in individual patients and groups undergoing different drug treatments. We compare time-lapse maps of $\mathrm{AD}, \mathrm{MCI}$, and other dementias, correlate these changes with cognition, and relate them to similar time-lapse maps of childhood development, schizophrenia, and HIV-associated brain degeneration. Strengths and weaknesses of these different imaging measures for basic neuroscience and drug trials are discussed.

\section{Keywords}

MRI; Alzheimer's disease; aging; MCI; dementia; brain degeneration; PET 


\section{INTRODUCTION}

Alzheimer's disease (AD) is arguably the greatest threat to public health in the 21 st century. Dementia doubles in frequency every 5 years after the age of 60, afflicting $1 \%$ of 60-64year olds but $30-40 \%$ of those aged 85 years and older. ${ }^{1}$ With the size of the elderly population rising dramatically and incidence of dementia also increasing, there are clear warning signs of an approaching socioeconomic disaster. As novel, disease-modifying agents emerge, brain imaging measures are vital to help demonstrate the effectiveness of pharmacologic treatments evidenced by any slowing of disease progression in the brain. Brain imaging measures facilitate drug development in animal models and patient studies, 2,3 and are also emerging as important tools for differential diagnosis of dementia. ${ }^{4}$

MRI and PET studies allow visualization of brain structure and function in threedimensional detail. When performed repeatedly over time, they can be used to visualize disease progression in living patients. Based on data from many subjects, measures of regional brain volumes and rates of atrophy may eventually aid in predicting who will suffer cognitive decline among those at risk for dementia. These measures are already being used to gauge the effects of therapy in drug trials. Imaging can also be used to track how different degenerative diseases spread in the living brain, providing a better theoretical understanding of how the various types of dementia differ (such as Alzheimer's disease vs. vascular or semantic dementia). Imaging can also be used to explore how the brain changes with normal aging, ${ }^{5}$ pinpointing changes associated with specific behavioral alterations, such as apathy or declining executive function. ${ }^{6}$

Brain imaging is also revealing important new information on other degenerative dementias, such as that resulting from HIV infection. Forty million people are infected with HIV worldwide, and at least $40 \%$ of these patients suffer from cognitive impairments ranging from minor cognitive motor disorders (MCMD) to HIV-associated dementia, often with a progressive trajectory leading to death. The trajectory of brain degeneration in HIV is markedly different from that seen in Alzheimer's disease-the caudate, white matter, and cortex degenerate progressively in a sequence that was recently visualized for the first time..$^{7-10}$ As in Alzheimer's disease, maps of degeneration based on MRI scans are increasingly needed to help gauge the success of neuroprotective therapies (e.g., memantine, CPI-1189, or NMDA-receptor antagonists in neuro-AIDS). As we describe later, MRI may also be combined with image analysis methods to track disease progression in individual patients.

Our understanding of degenerative disease has grown considerably due to rapid advances in brain imaging technologies. When combined with sophisticated analysis methods, structural MRI can now detect subtle, systematic brain volume changes on the order of $0.5 \%$ per year in individuals. ${ }^{3,11}$ If the same group of subjects is scanned repeatedly as their disease progresses, the dynamic trajectory of cortical atrophy can be reconstructed as it spreads over time in the living brain. ${ }^{12}$ New PET tracer compounds are being developed to visualize the profile of accumulating pathology ${ }^{13-17}$ Hailed as a breakthrough in the AD research community, these PET tracers visualize amyloid plaques and neurofibrillary tangles (NFT) in the living brain - hallmarks of AD previously only detectable at autopsy. These molecular probes are labeled with positron emitting isotopes-when they bind to the hallmark lesions of $\mathrm{AD}$, their distribution in the brain can be determined using PET scanning. Both the UCLA and Pittsburgh compounds-respectively called $\left[{ }^{18} \mathrm{~F}\right]$-FDDNP and $\left[{ }^{11} \mathrm{C}\right]-\mathrm{PIB}$ (Pittsburgh compound B)—show the expected pattern of accumulating pathology in initial studies of patients with $\mathrm{AD}$ and in small samples of mild cognitive impairment (MCI) subjects. ${ }^{15,17}$ As PET and MRI measures track different aspects of the disease process, there is a race to determine which imaging measures are most sensitive to disease progression, 
which techniques are most accurate in predicting imminent degenerative changes, and which are best at discriminating pathological from healthy aging. Clearly, techniques with a relatively high diagnostic specificity (such as cerebro-spinal fluid [CSF] measures of $\beta$ amyloid levels) may not offer the greatest sensitivity to disease progression over time. The pace of analytic developments in computational anatomy is also high. Newer computational methods, such as tensor-based morphometry (TBM), can detect increasingly subtle brain changes in conventional MRI scans, and newer imaging techniques, such as diffusion tensor imaging (DTI), are providing new insight into the pattern of deteriorating fiber architecture.

\section{Imaging of Alzheimer's Disease and MCl}

Imaging studies have become a priority in Alzheimer's disease research as they can be used to evaluate treatments that may slow or delay the disease process. ${ }^{2,3}$ A major practical goal is shortening the minimum feasible follow-up interval in a drug trial, or reducing the sample size required to detect a given degree of slowing of brain degeneration. This would have immediate practical advantages as new drugs could be tested much more efficiently. Clinical testing is commonly combined with neuroimaging to determine how best to identify presymptomatic candidates for preventive treatments before the extensive neuronal damage of $\mathrm{AD}$ has set in. MCI, for example, is a transitional state between normal aging and dementia. MCI subjects have a cognitive complaint and test 1.5 standard deviations below age- and education-adjusted norms on one or more neuropsychological tests, but they are still capable of independent living. ${ }^{18,19} \mathrm{MCI}$ is defined using neuropsychiatric criteria, but many brain imaging studies aim to develop measures that are sensitive enough to distinguish MCI from healthy aging with high specificity. ${ }^{20}$ Other studies attempt to differentiate between MCI subjects who will imminently convert to $\mathrm{AD}$, over a specific follow-up interval, versus those who remain stable or even recover. ${ }^{21,22}$

Scientific interest in MCI is rising as MCI subjects convert to full-blown AD at a rate 3-6 times higher than normal subjects. ${ }^{18,19}$ Alarmingly, post mortem studies have shown that most patients with MCI already have the pathological hallmarks of AD—neocortical senile plaques, NFT, atrophy, and neuronal loss in layer II of the entorhinal cortex. ${ }^{23,24}$ Many imaging studies target individuals with MCI as they are more likely than the general elderly population to obtain diagnoses of $\mathrm{AD}$ later in life. Research efforts may ultimately be of greater benefit to those with MCI given that early intervention may eventually prevent progression to global cognitive decline. As the risk of $\mathrm{AD}$ increases exponentially with age, delaying disease onset, even by a couple of years, would vastly reduce the overall number of cases of $\mathrm{AD}$.

\section{Statistical Power}

Improved treatment is needed for patients at all stages of $\mathrm{AD}$, but many drug trials focus on $\mathrm{MCI}$ subjects for pragmatic reasons. Cognitively normal elderly subjects convert to $\mathrm{AD}$ at a rate of only 1-2\% per year, so trials to resist conversion from normality to $\mathrm{MCI}$ or $\mathrm{AD}$ would need to follow 3,000-6,000 subjects for 5 to 7 years to achieve sufficient clinical endpoints. ${ }^{2}$ This is prohibitively expensive and too slow to be practical. On the other hand, MCI subjects convert to $\mathrm{AD}$ at a rate of $12 \%-15 \%$ per year. Secondary prevention studies (to prevent conversion from MCI to AD) typically need to assess only several hundred subjects, as a large proportion will convert to AD in 1-4 years (the duration of a typical research study). Even so, long time frames are necessary because of the high variability in clinical endpoints, and the relatively small degenerative changes that are barely detectable in brain images unless data from large numbers of subjects are combined (see Ref. 25, for studies of the detection limits of sequential MRI). 
If any imaging method could demonstrate slowing, in a quantitative measure of the disease process, the hurdle for treatments to pass would be greatly lowered-drug evaluation would be greatly accelerated. For example, a treatment would be regarded as promising if it were shown to slow whole brain atrophic rates, in MCI, by as little as $10 \%$ over a 6-month interval. Many more clinical trials would be attempted, as the time required, costs, and associated economic risks, would be greatly reduced. With this in mind, developers of computational techniques now aim to extract the maximum amount of information from images of disease progression, often by compiling population-based repositories of statistical data on expected rates of atrophy. Other efforts are mathematically distilling new sources of contrast from images (using new tracer kinetic models for PET, or combining scans acquired at different magnetic field strengths to detect new features, such as iron (ferritin) accumulation in the brain. ${ }^{26,27}$

Here we review recent progress in the neuroimaging of dementia, focusing on structural brain mapping studies that track the disease as it spreads in the living brain. These imaging approaches are being used to map disease progression in individuals and populations, revealing group patterns of cortical thinning, gray and white matter atrophy, and shape changes in subcortical structures, such as the hippocampus. Functional and structural imaging methods may also be combined, relating anatomical deficits to plaque and tangle pathology observed post mortem or with new PET tracers. These brain mapping techniques show promise in identifying predictors of imminent decline and disease onset, which are valuable for identifying candidates for drug trials. An overarching goal of most brain mapping studies is to extract and analyze statistical data on the disease process and develop new mathematical methods to quantify how well treatments resist AD (see Ref. 12, for a review).

\section{THE TRAJECTORY OF ALZHEIMER'S DISEASE}

Recent imaging studies have successfully tracked the emergence and spread of Alzheimer's disease pathology in the living brain (see e.g., Ref. 28). AD pathology progresses in a known, stereotypical sequence (Fig. 1), whether it is tracked with MRI, PET, SPECT, or in post mortem histologic studies of patients at different stages of the disease.

The time-course of disease progression varies substantially among individuals with $\mathrm{AD}$, but neurofibrillary pathology typically starts in the transentorhinal cortex and quickly spreads to the entorhinal cortex before involving the hippocampus. ${ }^{29-31}$ This temporal pathology persists for several years ${ }^{11}$ before spreading cortically to engulf the rest of the temporal, frontal, and parietal lobes. ${ }^{28,30-36}$

Longitudinal 3D MRI scanning of groups of subjects can be used to map this process in detail. Time-lapse maps have been constructed from sequential brain MRI scans to reveal the anatomical sequence of cortical atrophy ${ }^{28}$ (see http://www.loni.ucla.edu/ thompson/AD_4D/dynamic.html for movies of disease progression, that can be viewed over the Internet). Cortical regions that myelinate first-and most heavily - in development are typically least vulnerable to AD pathology (e.g., primary sensorimotor and visual cortices). In contrast, NFT and neuropil threads in dendrites accumulate early in the late myelinating heteromodal association cortices, the posterior cingulate, and phylogenetically older limbic areas that remain highly plastic throughout life. $^{41}$

\section{Why Does AD Progress in This Sequence?}

It is not known why AD engulfs the brain in this limbic-to-frontal sequence. The sequence is well documented and is largely agreed upon by the AD research community. Essentially, the 
same degenerative sequence was observed post mortem, and in FDG-PET data, many years before MRI scanning had sufficient resolution to gauge how cortical atrophy progresses. More recently, PET ligands that track the molecular hallmarks of AD show that pathology accumulates in a similar spreading pattern. Cross-sectional studies at UCLA and the University of Pittsburgh, using the molecular probes [ $\left.{ }^{18} \mathrm{~F}\right]-\mathrm{FDDNP}$ and $\left[{ }^{11} \mathrm{C}\right]-\mathrm{PIB}$, suggest that amyloid plaques deposit sequentially in the brain, appearing first in cingulate cortex, progressing to temporal-parietal cortices and the caudate, and finally engulfing occipital and sensorimotor cortices. ${ }^{15,17}$ This sequence agrees very closely with the post mortem Braak maps, ${ }^{30}$ and with MRI-based maps of cortical degeneration. ${ }^{28}$

Why the changes occur in this sequence is the subject of debate. The term "retrogenesis" has been used to describe some of the regressive behavioral changes that occur in dementia, when behavior may regress to a form resembling childhood or infancy. The sequence of cortical atrophy in Alzheimer's disease is in some respects an "unraveling," or recapitulation in reverse, of the anatomical sequence of childhood brain maturation. Primary sensory areas myelinate first in early infancy, but offer greatest resistance to neurodegeneration, staying intact in late AD. We recently developed a time-lapse map of cortical maturation, based on serial brain MRI scans of 13 children aged 4 to 21 years, scanned every 2 years for 8 years ${ }^{42}$ (see http://www.loni.ucla.edu/ thompson/DEVEL/dynamic.html for videos of these changes). In these images, a shifting pattern of gray matter loss appeared first (around ages 4-8 years) in dorsal parietal and primary sensorimotor regions near the interhemispheric margin, spreading laterally and caudally into temporal cortices and anteriorly into dorsolateral prefrontal areas. As expected from post mortem studies of cerebral myelination, ${ }^{43,44}$ the first areas to mature were those with the most basic functions, such as processing the senses and movement. Areas involved in spatial orientation and language (parietal lobes) followed, around the age of puberty (11-13 years). Areas with more advanced functions - integrating information from the senses, reasoning and other "executive" functions (prefrontal cortex)—matured last (in late adolescence).

Phylogenetically older cortical areas matured earlier than the more recently evolving higherorder association cortices, which integrate information from earlier maturing cortex. Provocatively, the last brain regions to develop in childhood are among the first to degenerate in dementia; and the earliest developing brain regions-subserving vision and sensation-are spared until the very latest stages of AD. As such, the age-related degenerative process progresses from late- to earlier myelinating regions. ${ }^{43,44}$

Bartzokis et al. ${ }^{50}$ examined this myelination sequence and suggested that late myelinating regions are especially vulnerable in $\mathrm{AD}$ and other adult onset disorders, such as schizophrenia. In support of this, the frontal cortex in schizophrenia has been found to undergo derailed maturation in patients and in at-risk relatives who subsequently convert to full-blown psychosis. ${ }^{45-48}$ If this is true, the anatomical trajectory of white matter maturation and degeneration has been comparatively overlooked in neuroimaging research. Recent work, with DTI, appears to support this view. In Kochunov et al. ${ }^{143}$ we found that the most prominent age-related drop in diffusion anisotropy occurred in late myelinating white matter, such as the genu of the corpus callosum, which innervates the frontal lobes. The cognitive consequences of this drop need to be established, but this is consistent with prior reports that late myelinating regions are most vulnerable to age-related degeneration. ${ }^{49-51}$

\section{Other Dementias}

Cortical changes do not follow the same trajectory in Alzheimer's disease as in other neurodegenerative diseases, or even other forms of dementia. In a recent study of Lewy body dementia (LBD), for example, we found that cortical gray matter in temporal and orbitofrontal cortices was comparatively preserved in 16 LBD patients (age: $76.4 \pm 6.7$ 
semantic dementia [SD]), compared with $29 \mathrm{AD}$ patients matched for age and dementia severity. LBD patients showed severe anterior cingulate atrophy compared to 38 matched controls. ${ }^{52}$ This was somewhat expected based on earlier neuropsychological studies of LBD, which suggested that temporal lobe degeneration and memory decline appear relatively late in LBD..$^{53,54}$

We also compared the 3D profiles of cortical atrophy in frontotemporal dementia (FTD) and SD. ${ }^{6}$ SD patients typically present with impoverished speech, but FTD patients show lack of empathy, disinhibition, and poor judgment. Congruent with these findings, we found predominantly posterior, left-sided gray matter $(\mathrm{GM})$ atrophy in $\mathrm{SD}(N=5)$ and more frontal, right-sided GM atrophy in FTD $(N=10)$. These studies, and others like them, suggest that pathophysiological differences underlying different forms of dementia are evident in brain images. Differential diagnosis based on MRI and PET is an active field. ${ }^{4}$

\section{Neurodegeneration in HIV/AIDS}

Perhaps the most startling finding in comparing degenerative patterns with MRI was the observation of selective cortical neurodegeneration in patients with HIV/AIDS, with greatest deficits in sensorimotor and supplementary motor cortices, and in the underlying white matter, basal ganglia, and hippocampus (Fig. 2). ${ }^{7,9}$

The resulting maps provide a new approach to gauge the impact of HIV on the living brain (see also Refs. 9,10, for related maps of white matter degeneration). This unusual degenerative pattern is the opposite of that seen in common noninfectious dementias, such as $\mathrm{AD}$, where the medial temporal, limbic, and association cortices are affected first, and primary sensorimotor and visual cortices only later. Clearly, different degenerative disorders may have quite different stereotypical patterns of atrophy and progression. Some have suggested the HIV virus migrates from the ventricles through the white matter to the cortex, ${ }^{55}$ where HIV virus-encoded proteins overactivate NMDA-type glutamate receptors and the excess extracellular glutamate causes excitotoxic injury and cell death. Why specific brain systems are especially vulnerable to the neurotoxic effects of HIV is not yet understood, but brain mapping is providing vital information on the trajectory of the disease.

\section{Association with Specific Symptoms}

If different forms of dementia progress in different ways, it is legitimate to ask whether different patterns of symptoms and functional decline are associated with different patterns of atrophy. In $\mathrm{AD}$, we found that distinct cortical atrophy patterns were associated with apathy, versus language dysfunction, or versus global cognitive decline. ${ }^{21,56,57}$ In a language study, we computed an individual average language domain $Z$-score from each subject's Z-scores on the Boston Naming Test and Animal Fluency test. Three-dimensional statistical maps then revealed that language performance was associated most strongly with left-sided gray matter loss in $19 \mathrm{AD}$ and $5 \mathrm{MCI}$ patients. More of the left hemisphere showed atrophy that linked with language function, suggesting that language abilities in AD are strongly influenced by cortical integrity in left perisylvian areas. A related study of healthy developing children revealed that thickening of language-related cortices, but not motor cortices, was linked with improvements in language function, and thickening of motor cortices, but not language cortices, was linked with improvements in motor function. ${ }^{58}$ This type of double dissociation is remarkable, as a more simplistic model might predict that all functional domains would improve somewhat equally in concert with a generalized maturation of the cortex. In healthy adults, the quantity of gray matter overall and especially in the frontal lobes is associated with better performance on standardized IQ tests, and both of these measures are under strong genetic control. ${ }^{59,60}$ This suggests a moderately strong linkage between regional brain volumes and intellectual ability. The association between 
brain volume and IQ has been replicated in numerous meta-analyses (e.g., Ref. 61), and may contribute to any detected association between cognitive performance and brain volumes in degenerative disease as well.

Mini-Mental State Exam (MMSE) scores offer a more general measure of overall cognitive decline in $\mathrm{AD}$, and declining MMSE is associated with widely distributed atrophy in both hemispheres. ${ }^{21,28}$ Anterior cingulate and supplementary motor cortices were more atrophied in $18 \mathrm{AD}$ patients with apathy versus 18 without apathy but matched for dementia severity. ${ }^{57}$ We also studied late-life depression in nondemented controls. ${ }^{62} \mathrm{We}$ found a regionally specific 5-20\% gray matter deficit in the orbitofrontal cortex of 24 depressed patients compared to 19 age-matched nondepressed subjects. In a collaboration with the University of Brescia, ${ }^{63}$ we compared cortical thinning in 10 late onset and 10 early onset AD patients of similar clinical severity (MMSE: $18.6 \pm 3.5$ vs. $19.2 \pm 3.5$ ). Early onset was defined as diagnosis before 65 years of age. Early onset AD primarily affected temporoparietal areas and late onset $\mathrm{AD}$ affected the medial temporal gray matter. These results may explain why early onset $\mathrm{AD}$ features primarily neocortical symptoms, while late onset $\mathrm{AD}$ generally features medial temporal symptoms.

These studies zero in on abnormal cortical systems contributing to specific functional deficits-revealing pathophysiological differences underlying the different symptoms of dementia or dementias with differences in the age of onset.

\section{METHODS TO TRACK BRAIN CHANGES IN ALZHEIMER'S DISEASE}

Methods to track structural brain changes with conventional MRI fall into three main categories: (1) volumetric measurement of specific structures, such as the hippocampus or entorhinal cortex; (2) image processing techniques that estimate rates of whole brain atrophy as a percentage volume loss per year ${ }^{11,64}$; and (3) map-based techniques that visualize the $3 \mathrm{D}$ profile of group differences in gray matter loss ${ }^{65}$ atrophic rates, ${ }^{66,67}$ white matter integrity, ${ }^{68}$ or cortical gray matter thickness. ${ }^{7,51,69,70}$ The main measures used in drug trials today are typically simpler ones that produce single numeric measures of disease burden, such as total hippocampal volume, or whole brain atrophic rates, which can all be expressed in cubic centimeters per year (or as a percentage change per year). The more exotic techniques that produce 3D maps of degenerative changes (reviewed below) have yielded substantial neuroscientific information on the disease trajectory, but have yet to gain acceptance in drug trials. Drug trials have not yet used these measures partly due to their complexity but also due to the pressure to express outcomes in a simple way, ideally using a small number of summary measures. Large-scale neuroimaging efforts (e.g., the Alzheimer's Disease Neuroimaging Initiative, www.loni.ucla.edu/ADNI) are now comparing the power of these map-based imaging measures, together with biomarkers and other clinical/functional measures, to differentiate MCI and AD from healthy aging, to predict future cognitive decline, and to predict conversion from MCI to AD. This is a vital effort—currently, given that in individual subjects assessed with MRI, MCI is not readily distinguished from $\mathrm{AD}$ or from normal aging, except when groups of subjects are compared in aggregate-individuals in each of these categories overlap substantially for all known MRI measures.

\section{Hippocampal Volumes and Maps}

$\mathrm{AD}$ pathology emerges first in the entorhinal cortex and hippocampus, so most volumetric MRI studies of MCI and AD patients have focused on the medial temporal lobe structures. Neuronal atrophy, decreased synaptic density, and overt neuronal loss is evident on MRI as progressive cortical gray matter loss, reduced subcortical gray and white matter volumes, and expanding ventricular and sulcal CSF spaces. ${ }^{71}$ 
Patients with mild AD have roughly $25 \%$ smaller hippocampal volumes than matched healthy elderly controls, ${ }^{72-74}$ whereas MCI patients show a mean reduction of around $11 \% .^{74}$ Early studies by Jack et al. ${ }^{144}$ found that $97.2 \%$ of the patients studied with very mild AD - that is, with Clinical Dementia Rating 0.5-had hippocampal volumes below the healthy normal average. In MCI, mean hippocampal volumes are variously reported as being roughly half way between $\mathrm{AD}$ and normals, ${ }^{75-78}$ or as being more similar to AD patients. ${ }^{79,80} \mathrm{Xu}$ et al. ${ }^{145}$ found that entorhinal cortex and hippocampal volume measures provided roughly equal intergroup discrimination ability in 30 control, $30 \mathrm{MCI}$, and $30 \mathrm{AD}$ subjects; even though the entorhinal cortex typically atrophies earlier, it is harder to delineate reliably on MRI. Many groups have also used premorbid hippocampal atrophy, rated visually ${ }^{81}$ or using volumetry, ${ }^{82-84}$ to predict subsequent crossover to AD. Jack et $a{ }^{76}$ also found that hippocampal atrophy at baseline was associated with conversion from MCI to AD at a 33-month follow-up (relative risk: $0.69, P=0.01 ; 27$ of $80 \mathrm{MCI}$ subjects had become demented).

Three-dimensional modeling techniques (Fig. 3) have localized specific regions of atrophy within the hippocampus in MCI. For example, Becker et al. ${ }^{20}$ used3Dsurface reconstruction techniques to model the shape of the hippocampus, creating average shape models for cohorts of subjects with amnestic MCI, nonamnestic MCI, AD, and healthy controls. These techniques can localize tissue atrophy or shape alterations ${ }^{85}$ and can map the average pattern of hippocampal thickness reductions in millimeters. ${ }^{71}$ Amnestic MCI patients showed diffuse hippocampal atrophy, ${ }^{86}$ and reduced volumes in the mesial temporal lobe including the hippocampus, entorhinal cortex, and amygdala. ${ }^{87}$ Nonamnestic MCI patients-who show cognitive impairments in single or multiple domains other than memory-typically have greater atrophy outside of the hippocampus, specifically in multimodal association cortices.

These methods provide, at each location on the hippocampal surface, a measure of how much radial atrophy there is, as either a proportion of average control values, or as a $Z$-score, or a significance map comparing one group with another. These methods have been used to detect structural differences in the hippocampus that are associated with Alzheimer's disease, ${ }^{71,88} \mathrm{MCI},{ }^{20,21}$ schizophrenia, ${ }^{89}$ normal development, ${ }^{90}$ methamphetamine abuse, ${ }^{91}$ epilepsy, ${ }^{92}$ bipolar illness, ${ }^{93}$ depression, ${ }^{94}$ and HIV/AIDS. ${ }^{86}$ Frisoni et al. ${ }^{88}$ also used this method to compare a group of 28 AD patients and 40 elderly controls, and found significant tissue loss (20\% or more) in the hippocampal CA1 fields and part of the subiculum. As expected from pathological studies, ${ }^{95}$ regions corresponding to the CA2-3 fields were relatively spared. This atrophy distribution largely corresponds to the known selective involvement of hippocampal regions by AD pathology, and supports the possibility of carrying out in vivo macroscopic neuropathology of the hippocampus with MR imaging in the dementias.

Predicting Outcomes in $\mathbf{M C l}-$ Hippocampal surface maps have also been used to predict outcomes in MCI. In 20 MCI subjects followed neuropsychologically for 3 years, maps of hippocampal atrophy at the start of the study successfully differentiated the 7 patients who later converted to AD from 6 who stayed stable and 7 who improved. CA1 and subicular degeneration were associated with conversion to $\mathrm{AD}$; these areas were intact in MCI patients who improved and no longer met MCI criteria at follow-up. ${ }^{21}$

Accelerated Hippocampal Atrophy in Those at Genetic Risk-The same hippocampal mapping technique has been used to detect accelerated atrophy in healthy elderly people at genetic risk for Alzheimer's disease. In a longitudinal study, ${ }^{96}$ 3D hippocampal surface models were generated from volumetric brain MRI scans of 54 subjects (27 men, 27women), scanned at two different time points (mean age at baseline $=$ 
68.6, mean interval: 1.6 years, 19 ApoE4 carriers: 2 homozygous, 17 heterozygous). We found a strong correlation $(P=0.024)$ between the left hippocampal rate of atrophy and the ApoE genotype, with E4 carriers showing a greater average atrophic rate than non-E4 carriers (left hippocampus:-2.4\%/year vs. 0.36\%/year). Progressive hippocampal head atrophy occurred in healthy ApoE4 carriers, an atrophic pattern that is accelerated in AD. The maps showing greater average annualized loss and faster left hippocampal atrophy for ApoE4 carriers indicate that, in those at genetic risk for $\mathrm{AD}$, abnormally accelerated structural changes are detectable prior to cognitive decline.

\section{Automated Mapping of Gray Matter Changes: Voxel-Based Morphometry}

Conventional region-of-interest approaches, which use manual tracing to determine the volume of the structures, are ubiquitous but are gradually being replaced by more automated techniques for rapid large-scale processing of scans (see Ref. 97, for a review). ${ }^{25,98}$

Automated image registration approaches can align groups of images into a common space and intergroup differences can be assessed using voxel-by-voxel statistics. Threedimensional statistical maps of group differences in brain structure can be visualized, identifying regions where atrophy correlates with diagnosis, as well as clinical, therapeutic, genetic, or functional measures. Chetelat et al. ${ }^{146}$ used an automated technique known as voxel-based morphometry (VBM) ${ }^{99,100}$ to map gray matter changes in 18 amnestic MCI patients. In a follow-up scan 18 months later, subjects who had converted to Alzheimer's disease showed significantly greater GM loss- relative to nonconverters-in the hippocampus, inferior and middle temporal gyri, posterior cingulate, and precuneus. All of these regions show severe deficits in mild $\mathrm{AD}$ (see Fig. 1).

\section{Optimized VBM}

Brief comment is warranted regarding a controversy that arose in the literature regarding the use of VBM. ${ }^{100-104}$ This debate generated some confusion regarding the validity of VBM. VBM is an extremely popular approach for comparing structural brain images, partly due to its high automation, and it is implemented in the Statistical Parametric Mapping image analysis package (SPM; www.fil.ion.ucl.ac.uk/spm/). Prior to 2000, the most commonly used implementation of VBM involved warping all tissue class images (i.e., 3D maps of gray and white matter probabilities) to match the same standardized brain atlas, and preserving the probability values in the spatially transformed images. These data were typically then smoothed and statistical analyses performed. This approach was criticized by Bookstein $^{101}$ and Thacker ${ }^{103}$ who noted that this method only detects registration errors, because if the warping was $100 \%$ exact, there would be no residual differences. Bookstein noted that the results of such studies depend heavily on the registration strategy used, and therefore VBM should not be used. In response to this criticism, the authors of VBM developed a modified approach, known as "modulated VBM."100 This approach warps the tissue class images, but preserves information on the total volume of tissue by scaling the image intensities according to the amount of expansion or contraction. This approach was independently suggested by Davatzikos et al. ${ }^{102}$ where it was termed "RAVENS" (Regional Analysis of Volumes Examined in Normalized Space); the same approach is known as "modulated VBM" by SPM users. Here the comparisons are volumetric, and the resulting "optimized" VBM approach is now similar in many respects to the "tensor-based morphometry" methods described below.

\section{Surface-Based Analysis of Cortical Thickness}

Cortical modeling ${ }^{12}$ may also be used to map the profile of gray matter thickness across the cortical mantle, providing better localization and statistical power by matching data from corresponding gyri, as far as possible, across subjects. These cortical modeling approaches can be considered as a variant of VBM, but offer several benefits. First, parameters, such as 
cortical thickness, can be computed in 3D, displayed on the cortical models, and averaged across subjects for group comparisons. Second, the explicit modeling of the cortical surface geometry allows one cortex to be precisely aligned with another before multisubject comparisons are made. This greatly reduces registration errors when comparing data across subjects and groups. An additional step known as cortical pattern matching can be used to fluidly match the entire gyral pattern from subject to subject, using a 3D deformation field to drive sulcal landmarks into correspondence. ${ }^{12}$ This very high-order matching of anatomy can improve the power to detect subtle gray matter differences between groups or over time, and localizes effects relative to known cortical landmarks. In this process, clear anatomic divisions often emerge in the regions affected (e.g., the degree of cortical degeneration differs substantially on either side of the cingulate sulcus in (Fig. $1 \mathrm{C}$ ), a feature corroborated by the post mortem maps).

Composite maps of cortical thickness, based on MRI, have revealed a complex shifting pattern of cortical atrophy over the human life span, which is thought to primarily reflect neuronal shrinkage rather than overt neuronal loss $\left(N=176^{105} ; N=106\right.$ subjects $\left.^{51}\right)$. These cortical mapping techniques show great promise, but have yet to be applied in MCI.

Cortical gray matter thickness is a powerful neuroimaging marker of cortical integrity, and a reasonable question is: "What exactly is it measuring?" It is sensitive to subtle diseaserelated changes, and correlates with cognitive decline in Alzheimer's disease and schizophrenia. ${ }^{28,45}$ Cortical thickness on MRI may be related to regional neuronal density in the cortical mantle, ${ }^{106}$ but it may also depend on unknown vascular factors, glial cell numbers, and the extent and integrity of cortical myelination. We recently developed an approach to measure the thickness of the cortex from MRI, and mapped how it changes over the human life span. This approach has now been used in more than 30 studies, to compile composite maps of average cortical thickness and compare them between specific populations (see Ref. 12 for an overview; Fig. 4 shows the main steps). Regions with systematic differences in cortical thickness can be detected and displayed on the cortex, as can the trajectory of changes in development or disease.

Cortical thickness can be defined in several different ways, but the simplest approach computes the 3D distance from the inner cortical gray-white matter boundary in tissueclassified brain volumes to the outer cortical surface (gray-CSF boundary) in each subject. ${ }^{12}$ Neuroimaging and histological studies have found significant changes in cortical thickness in normal subjects. ${ }^{5,42,107,108}$ In one study, we examined a large sample $(N=176)$ of normal individuals across the span of life between 7 and 87 years. ${ }^{5,70}$ Cortical thickness decreased quadratically with age from an average thickness of $\sim 2.6 \mathrm{~mm}$ at 20 years to less than $2 \mathrm{~mm}$ in the age range 80-90 years. Perhaps surprisingly, scatterplots of these effects revealed a dramatic decline in gray matter density between the ages of 7 and 60 years with a slower decline thereafter, in most brain regions (the most dramatic changes occurring in the frontal cortex during late adolescence, where gray matter thickness falls rapidly). A second surprise was that the most lateral aspects of the brain in the posterior temporal and inferior parietal lobes bilaterally showed a distinct pattern of gray matter change, one in which the nonlinear age effects were inverted relative to the age effects seen in more dorsal cortices, that is, cortical atrophy accelerated rather than remaining linear or slowing down with age.

Gender differences in other markers of cortical atrophy are also apparent. In Kochunov et $a l .,{ }^{147}$ we studied age-related trends for the width and the depth of major cortical sulci in 90 healthy subjects ( 47 males, 43 females) aged $20-82$ years. The average sulcal width increased by $\sim 0.7 \mathrm{~mm} /$ decade, while the average sulcal depth decreased by $\sim 0.4 \mathrm{~mm} / \mathrm{dec}$ ade. Greater age-related decline was found in men relative to women, and in multimodal relative to unimodal cortical areas. 
The rate of cortical thickness reduction in abnormal aging and degenerative disorders has also been shown to be different from that in healthy aging, making it a useful biomarker of neurodegeneration in a variety of illnesses. ${ }^{12,28,112}$ Our cortical mapping approach has been used to detect unsuspected alterations in gray matter distribution in Alzheimer's disease, ${ }^{21,28,63}$ LBD $,{ }^{52} \mathrm{MCI},{ }^{21}$ late-life depression, ${ }^{62} \mathrm{HIV} / \mathrm{AIDS},{ }^{7}$ methamphetamine users, ${ }^{75}$ childhood and adult onset schizophrenia, ${ }^{45,110,113}$ normally developing children ${ }^{42,114,115}$ fetal alcohol syndrome, ${ }^{116}$ individuals at risk for schizophrenia, ${ }^{48}$ adult and adolescent onset bipolar illness, ${ }^{93,117}$ velocardiofacial syndrome $\left(\mathrm{VCFS}^{118}\right)$, Williams syndrome,${ }^{8}$ epilepsy, ${ }^{119}$ attention deficit hyperactivity disorder, ${ }^{105}$ genetic influences on brain structure, ${ }^{59,120}$ and lithium effects on brain structure. ${ }^{93}$ The most general review of these methods is in Thompson et al. ${ }^{12}$

One of the most provocative recent findings is that significant cortical thinning is detectable in asymptomatic carriers of the high-risk allelic variant, apolipoprotein E $\varepsilon 4$ (APOE $\varepsilon 4$ ). This allele is quite prevalent in the general population, and results in a threefold increased risk of developing Alzheimer's disease. Using a technique to unfold the convoluted geometry of the hippocampus, ${ }^{121}$ Burggen et al. ${ }^{148}$ found that 9 (heterozygous) APOE $\varepsilon 4$ carriers with normal memory performance showed significantly reduced cortical thickness compared to 14 noncarriers in entorhinal cortex and the subiculum (by $10.6 \%$ and $7.5 \%$ respectively; $P=0.005,0.003$ ), but not in the main body of the hippocampus or perirhinal cortex. Such a pattern of cortical thinning is consistent with the known progression of AD pathology and may reflect either a preexisting reduction in cortical thickness, or early dynamic changes in cortical structure that diminish the processing capacity of the cortex. These early changes may contribute to accelerated disease onset.

\section{Imaging White Matter with DTI}

White matter changes in MCI and AD are also of interest. Conventional MRI has insufficient contrast to discriminate fiber tract organization within the white matter, but DTI, a newer MRI variant introduced in the mid 1990s, ${ }^{122-124}$ is sensitive to myelin breakdown, as well as fiber integrity and orientation. Medina et al. ${ }^{68}$ found that groups of MCI and AD subjects showed abnormal reductions in fractional anisotropy, a DTI-based measure of fiber integrity, in multiple posterior white matter regions. Rose et al. ${ }^{149}$ found that relative to normal controls, AD patients showed a significant reduction in the integrity of the association white matter fiber tracts, including the splenium of the corpus callosum, the superior longitudinal fasciculus, and cingulum but not the pyramidal tracts. This is consistent with the typical clinical presentation of $\mathrm{AD}$, that is, global cognitive decline but no motor disturbances. Other measures of water diffusion, such as the apparent diffusion coefficient (ADC), are abnormally elevated in the hippocampus in $\mathrm{MCI},{ }^{125,126}$ and in broader areas-including the parietal white matter-in established AD. ${ }^{127}$ Abnormal white matter changes can therefore be detected in MCI, prior to the development of dementia.

\section{Tensor-Based Morphometry}

The best MRI measure to monitor disease progression in dementia depends, to some degree, on the follow-up interval after which patients are reassessed. At relatively short follow-up intervals (6 months), ventricular measures are three times more powerful than whole brain atrophic rates for distinguishing $\mathrm{AD}$ from controls; this advantage dissipates if the follow-up interval is extended to 1 year. ${ }^{128}$

Demonstration of deteriorating brain structure during the transition from MCI to AD has significant prognostic value as such changes over time almost certainly reflect the progression of underlying brain pathology. For tracking brain changes in exquisite detail, tensor-based morphometry (TBM) - also known as deformation morphometry or voxel 
compression mapping - has emerged as a powerful method to track brain change (Thompson et al.; Fox et al.; Janke et al.; Studholme et al.; Leow et al.; Chiang et al. $)^{25,66-132}$ TBM is a more complex image analysis approach; it quantifies tissue growth or atrophy throughout the brain, based on elastically warping sequentially collected MRI scans. It visually indicates the local rate at which tissue is being lost, or expanding, throughout the anatomy of the brain (see Fig. 5 for an illustration). It surveys the whole brain at once, but detects localized changes occurring at a regional level, without having to specify the regions of interest in advance. This approach may ultimately offer the greatest power for clinical trials. Deformation morphometry can detect subtle medication-related changes over a period of less than a month, such as effects of lithium on brain structure, ${ }^{133}$ and is automated enough to apply to large cohorts of subjects efficiently, without the need for laborious interaction with images to identify regions of interest. The brain changes are then compared across subjects or groups. Correlations can be mapped between these changes and demographic or clinical measures, such as age, sex, diagnosis, cognitive scores, treatment outcomes, or biological serum measures (see Ref. 9, for examples of this approach in a study of HIV/AIDS).

How TBM Detects Brain Changes-If two brain images, acquired over time, are rigidly overlaid, brain changes cannot be localized: no information is available on exactly where atrophy is occurring. To localize changes within the brain, matching needs to be performed with a nonlinear "warping" algorithm. Over the last 15 years, many groups have developed nonlinear image warping techniques that align brain images with a compressible elastic or fluid model. This allows localized deformation. How well these approaches perform depends on the mathematical measure of image correspondence used to drive the alignment of the images, how flexible the deformation is (in terms of degrees of freedom), and whether steps are taken to ensure that the mappings are smooth and preserve the topology of the image. ${ }^{134}$ In one approach (see Refs. 67,132, for mathematical details), the follow-up (repeat) image is globally aligned to the baseline scan, and then a 3Delastic or fluid image deformation is used to maximize the mutual information (or a related information-theoretic measure of correspondence) between the two consecutive scans. This fully $3 \mathrm{D}$ deformation reconfigures the baseline anatomy into the shape of the follow-up scan. The expansion or contraction at each image voxel is computed from the deformation field (using the Jacobian of the deformation field to produce a "voxel compression map" or "tensor map"). In this map, contraction implies atrophy; expansion implies local growth or dilation. ${ }^{64} \mathrm{~A}$ color map then displays these changes on the follow-up scan. As shown in Figure 3, the method can be used to map patterns of brain changes in patients scanned longitudinally over short intervals, ${ }^{25}$ in patients with semantic dementia, ${ }^{135}$ and has been used to automatically map the profile of brain structural differences in cohorts with HIV/ AIDS, ${ }^{9,10}$ Fragile $\mathrm{X}$ syndrome, ${ }^{136}$ Williams syndrome, ${ }^{137}$ schizophrenia, ${ }^{58}$ bipolar illness, ${ }^{138}$ in healthy subjects treated with lithium, ${ }^{133}$ and in twins imaged with DTI. ${ }^{139}$ TBM has a relatively high throughput and sensitivity, making it attractive to use for gauging brain changes in large population studies and clinical trials. New statistical methods are also emerging to increase the sensitivity of TBM. For detecting degenerative brain changes, Lie group and Riemannian manifold methods can provide more statistical power than standard approaches, as they draw upon the full multidimensional information available in the deformation tensors. ${ }^{10,139}$ Such an approach might be termed "generalized TBM."

\section{Combining Fluid Registration with Anatomical Surface Modeling}

Fluid registration can also be combined with surface-based modeling to automatically create maps of anatomical surfaces in large numbers of images, and compare the resulting surfaces statistically. This approach combines the strengths of two very different methods, while providing automation and high throughput for large population studies. In the largest brain 
mapping study of MCI to date, Carmichael et al..$^{22}$ delineated the lateral ventricles on a single brain MRI data set, and fluidly deformed a surface model of these structures, using nonlinear image registration, to match the shape of the ventricles in other MRI scans from $74 \mathrm{MCI}, 225$ normal, and $40 \mathrm{AD}$ subjects. The average ventricular shapes were compared using a surface-based modeling approach, which plots the regions with statistical shape differences on the surfaces using a color code. ${ }^{12}$ MCI subjects showed significant, localized dilations relative to normal subjects in the atrium of the ventricles and in the occipital horns. $\mathrm{AD}$ subjects showed a greater extent of enlargement involving the frontal horns. In $\mathrm{AD}$, temporal horn expansion progresses at a rate of $13-18 \%$ per year (compared with 2-4\%/year in healthy controls ${ }^{71}$ ). In contrast, the mean annual rate of hippocampal volume loss on MRI is much more subtle: $1.73 \%$ in stable controls, $3.5 \%$ in $\mathrm{AD}$, and $3.69 \%$ versus $2.55 \%$ in $\mathrm{MCI}$ subjects who decline or remain stable at 3-year follow-up. ${ }^{140}$ Strictly speaking, this approach provides similar information to TBM, but surface models are used to constrain the search for significant effects to regions known to be implicated. This improves statistical power, as well as presenting results in a visually intuitive way.

\section{USE OF IMAGING IN DRUG TRIALS}

Recent studies also suggest that imaging can be useful as a biomarker for therapeutic efficacy in $\mathrm{AD}$. Refinements are occurring in the measures used, and large-scale projects, such as the Alzheimer's Disease Neuroimaging Initiative (www.loni.ucla.edu/ADNI/) are now comparing and cross-validating different imaging measures for detecting significant brain changes in $\mathrm{AD}$. Even if only conventional volumetric measures are used, Jack et al. ${ }^{2}$ estimated that in each arm of a therapeutic trial, only 21 subjects would be required to detect a 50\% reduction in the rate of decline, if the hippocampal volume was used as the outcome measure. This compares with 241 subjects if MMSE scores were used, and 320 if the AD Assessment Scale Cognitive Subscale were used. Imaging measures are not likely to replace cognitive measures as outcome measures in clinical trials, but they are widely regarded as more stable and reproducible across sites. Significant work has been devoted to establishing imaging protocols that are reproducible across imaging centers and across time, ${ }^{141}$ and the detection limits of image analysis techniques have been shown to be greatly improved if scanner-related factors, such as geometric stability, field homogeneity, RF coil type, and field strength are optimized. ${ }^{25,141}$

Automated measures of atrophy are also gaining acceptance in longitudinal studies, although not all studies have yielded the expected conclusions. Fox et $a .^{3}$ applied a powerful image analysis approach, known as the Brain Boundary Shift Integral, to estimate the overall brain volume decrease in registered serial images from $288 \mathrm{AD}$ patients in a Phase IIa immunotherapy trial. Paradoxically, when assessed 11 months later, antibody responders $(N$ $=45$ ) had greater brain volume decreases (on average $3.1 \%$ vs. $2.0 \%$ ), and greater ventricular enlargement than placebo patients $(N=57)$. Because this atrophy did not correlate with cognitive decline, the authors speculated that these volume changes may be attributed to amyloid removal and associated cerebral fluid shifts.

\section{CONCLUSION}

Overall, structural brain scanning with MRI has yielded several measures that help to differentiate mildly impaired patients from controls. MRI can also be used to help predict who will imminently convert to suspected $\mathrm{AD}$, and to gauge how well interventions resist atrophic brain changes, if at all, in clinical trials. Because therapeutic trials require sensitive biomarkers that track the disease process in detail, serial MRI scanning is often combined with powerful, automated analysis methods to compute maps of brain changes, and volumetric measures for brain regions that change early in $\mathrm{AD}$, such as the hippocampus and 
entorhinal cortex. These efforts now use cutting-edge mathematics to map brain change on MRI and PET, as well as longitudinal data sets that track disease over multiyear time spans before and after disease onset.

Other innovations in neuroimaging include ongoing developments in DTI, MR relaxometry, imaging of iron deposition, and high-field MRI scanning. Each of these techniques aims to increase the repertoire of signals available for assessing tissue integrity in early neurodegenerative disease. Perhaps the most promising development is the recent advent of PET tracer compounds that visualize amyloid plaques and NFT in the living brain. These pathological features are the defining hallmarks of $\mathrm{AD}$, previously only detectable at autopsy. New tracer compounds for plaque and tangle imaging $\left(\left[{ }^{18} \mathrm{~F}\right]\right.$-FDDNP and $\left[{ }^{11} \mathrm{C}\right]-$ PIB) complement the now-standard PET measures (of perfusion and glucose metabolism) that are the mainstay of differential diagnosis in dementia. Many see in these new tracers the promise to track $\mathrm{AD}$ before it is clinically detectable — and assess how therapy resists it before symptoms become irreversible. Amyloid deposition gradually increases in the brain long before detectable symptoms of memory decline, so there is an expectation that amyloid plaque and/or tangle PET tracers can identify and monitor AD progression before any symptoms appear. ${ }^{15,17}$ In addition, these tracers quantify pathology objectively, offering a direct means to evaluate antiamyloid therapies, such as secretase inhibitors.

It is not yet known whether these new PET markers correlate more tightly than MRI measures with observable clinical decline in MCI, although both distinguish AD and MCI from controls. A major effort in these analyses is to correlate the trajectory of pathology observed with new PET tracers with dynamic maps of cortical neurodegeneration on MRI. Initial studies in $\mathrm{AD}$ reveal a positive correlation between rates of whole brain atrophy and regional $\left[{ }^{11} \mathrm{C}\right]$-PIB uptake $\left(N=9^{142}\right)$. Each of these measures is tightly correlated with global cognitive function (MMSE ${ }^{17,28}$ ). It is of interest to relate the two views of the neurodegenerative process. A major goal is to develop joint measures of the disease process with far greater predictive power than either MRI or PET can provide on its own.

\section{Acknowledgments}

This research was supported by the National Institute on Aging, the National Library of Medicine, the National Institute for Biomedical Imaging and Bioengineering, the National Center for Research Resources, and the National Institute for Child Health and Development (AG016570, LM05639, EB01651, RR019771, and HD050735 to P.M.T.). E.R.S. was supported by the National Institute of Drug Abuse (NIDAR21DA15878 and RO1DA017831 to E.R.S.). Additional support was provided by a Taiwan government fellowship (to M.C.C.), and NCRR grants RR13642 and RR021813 (to A.W.T.).

\section{REFERENCES}

1. Jorm AF, Korten AE, Henderson AS. The prevalence of dementia: a quantitative integration of the literature. Acta Psychiatr. Scand. 1987; 76:465-479. Review. [PubMed: 3324647]

2. Jack CR Jr, Slomkowski M, Gracon S, et al. MRI as a biomarker of disease progression in a therapeutic trial of milameline for AD. Neurology. 2003; 60:253-260. [PubMed: 12552040]

3. Fox NC, Black RS, Gilman S, et al. AN1792(QS-21)-201 Study 2005. Effects of Abeta immunization (AN1792) on MRI measures of cerebral volume in Alzheimer disease. Neurology. 64:1563-1572. [PubMed: 15883317]

4. Silverman DHS, Thompson PM. Structural and functional neuroimaging: focusing on mild cognitive impairment. Appl. Neurol. 2006; 2:10-24.

5. Sowell ER, Peterson BS, Thompson PM, et al. Mapping cortical change across the human lifespan. Nat. Neurosci. 2003; 6:309-315. [PubMed: 12548289]

6. Apostolova, LG.; Clark, DG.; Zoumalan, C., et al. 3D mapping of gray matter atrophy in semantic dementia and frontal variant frontotemporal dementia. International Conference on Alzheimer's Disease (ICAD2006); Madrid, Spain. 2006. 
7. Thompson PM, Dutton RA, Hayashi KM, et al. Thinning of the cerebral cortex in HIV/AIDS Reflects CD4 ${ }^{+}$T-lymphocyte decline. Proc. Natl. Acad. Sci. 2005; 102:15647-15652. [PubMed: 16227428]

8. Thompson PM, Lee AD, Dutton RA, et al. Abnormal cortical complexity and thickness profiles mapped in williams syndrome. J. Neurosci. 2005; 25:4146-4158. [PubMed: 15843618]

9. Chiang MC, Dutton RA, Hayashi KM, et al. Fluid registration of medical images using jensen-rényi divergence reveals 3D profile of brain atrophy in HIV/AIDS. IEEE Int. Symp. Biomed. Imag. (ISBI2006). 2006

10. Lepore, N.; Brun, CA.; Chou, YY., et al. Generalized tensor-based morphometry of HIV/AIDS using multivariate statistics on strain matrices and their application to HIV/AIDS. In: Gee, JC.; Thompson, PM., editors. submitted to IEEE Transactions on Medical Imaging Special Issue on Computational Neuroanatomy; 2006. to appear March 2007 [submitted, July 1, 2006]

11. Smith SM, Zhang Y, Jenkinson M, et al. Accurate, robust, and automated longitudinal and crosssectional brain change analysis. Neuroimage. 2002; 17:479-489. [PubMed: 12482100]

12. Thompson, PM.; Hayashi, KM.; Sowell, ER., et al. Mapping cortical change in Alzheimer's disease, brain development, and schizophrenia, Special Issue on Mathematics in Brain Imaging. In: Thompson, PM.; Miller, MI.; Ratnanather, JT.; Poldrack, R.; Nichols, TE., editors. NeuroImage. Vol. 23. 2004. p. S2-S18.

13. Barrio JR, Huang S-C, Cole GM, et al. PET imaging of tangles and plaques in Alzheimer disease. J. Nucl. Med. 1999; 40 Suppl:70P-71P. [PubMed: 9935060]

14. Shoghi-Jadid K, Small GW, Agdeppa ED, et al. Localization of neurofibrillary tangles and betaamyloid plaques in the brains of living patients with Alzheimer disease. Am. J. Geriatr. Psychiatry. 2002; 10:24-35. [PubMed: 11790632]

15. Klunk WE, Lopresti BJ, Ikonomovic MD, et al. Binding of the positron emission tomography tracer Pittsburgh compound-B reflects the amount of amyloid-beta in Alzheimer's disease brain but not in transgenic mouse brain. J. Neurosci. 2005; 25:10598-10660. [PubMed: 16291932]

16. Kepe V, Barrio JR, Huang SC, et al. Serotonin 1A receptors in the living brain of Alzheimer's disease patients. Proc. Natl. Acad. Sci. USA. 2006; 103:702-707. Epub 2006 Jan 9. [PubMed: 16407119]

17. Small GW, Kepe V, Ercoli L, et al. PET of brain amyloid and tau in mild cognitive impairment. N. Engl. J. Med. 2006; 355(25):2652-2663. [PubMed: 17182990]

18. Petersen RC, Smith GE, Waring SC, et al. Mild cognitive impairment: clinical characterization and outcome. Arch. Neurol. 1999; 56:303-308. [erratum appears in Arch Neurol 1999 Jun;56(6):760]. [PubMed: 10190820]

19. Petersen RC. Aging, mild cognitive impairment, and Alzheimer's disease. Neurol. Clin. 2000; 18:789-806. [PubMed: 11072261]

20. Becker JT, Davis SW, Hayashi KM, et al. 3D patterns of hippocampal atrophy in mild cognitive impairment. Arch. Neurol. 2006; 63:97-101. [PubMed: 16401741]

21. Apostolova LG, Steiner CA, Akopyan GG, et al. 3D gray matter atrophy mapping in mild cognitive impairment and mild Alzheimer's disease. 2007 [submitted].

22. Carmichael OT, Thompson PM, Dutton RA, et al. Mapping ventricular changes related to dementia and mild cognitive impairment in a large community-based cohort. IEEE Int. Symp. Biomed. Imag. (ISBI2006). 2006

23. Price JL, Morris JC. Tangles and plaques in nondemented aging and "preclinical" Alzheimer's disease. Ann. Neurol. 1999; 45:358-368. [PubMed: 10072051]

24. Kordower JH, Chu Y, Stebbins GT, et al. Loss and atrophy of layer II entorhinal cortex neurons in elderly people with mild cognitive impairment. Ann. Neurol. 2001; 49:202-213. [PubMed: 11220740]

25. Leow AD, Klunder AD, Jack CR, et al. Longitudinal stability of MRI for mapping brain change using tensor-based morphometry. Neuroimage. 2006; 31:627-640. Epub 2006 Feb 15. [PubMed: 16480900]

26. Bartzokis G, Sultzer D, Mintz J, et al. In vivo evaluation of brain iron in Alzheimer's disease and normal subjects using MRI. Biol.Psychiatry. 1994; 35:480-487. [PubMed: 8018799] 
27. Bartzokis G, Tishler TA, Lu PH, et al. Brain ferritin iron may influence age- and gender-related risks of neurodegeneration. Neurobiol. Aging. 2007; 28:414-423. [PubMed: 16563566]

28. Thompson PM, Hayashi KM, de Zubicaray G, et al. Dynamics of gray matter loss in Alzheimer's Disease. J. Neurosci. 2003; 23:994-1005. [PubMed: 12574429]

29. Braak H, Braak E. Neuropathological stageing of Alzheimer-related changes. Acta Neuropathol. 1991; 82:239-259. [PubMed: 1759558]

30. Braak H, Braak E. Staging of Alzheimer-related cortical destruction. Int. Psychogeriatr. 1997; 9 Suppl 1:257-261. discussion 269-272. [PubMed: 9447446]

31. Gomez-Isla T, Price JL, McKeel DW, et al. Profound loss of layer II entorhinal cortex neurons occurs in very mild Alzheimer's disease. J. Neurosci. 1996; 16:4491-4500. [PubMed: 8699259]

32. Frisoni GB, Laakso MP, Beltramello A, et al. Hippocampal and entorhinal cortex atrophy in frontotemporal dementia and Alzheimer's disease. Neurology. 1999; 52:91-100. [PubMed: 9921854]

33. Laakso MP, Partanen K, Riekkinen P, et al. Hippocampal volumes in Alzheimer's disease, Parkinson's disease, with and without dementia, and in vascular dementia: an MRI study. Neurology. 1996; 46:678-681. [PubMed: 8618666]

34. Laakso MP, Soininen H, Partanen K, et al. MRI of the hippocampus in Alzheimer's disease: sensitivity, specificity, and analysis of the incorrectly classified subjects. Neurobiol. Aging. 1998; 19:23-31. [PubMed: 9562499]

35. Dickerson BC, Goncharova I, Sullivan MP, et al. MRI-derived entorhinal and hippocampal atrophy in incipient and very mild Alzheimer's disease. Neurobiol. Aging. 2001; 22:747-754. [PubMed: 11705634]

36. Thal LJ. How to define treatment success using cholinesterase inhibitors. Int. J. Geriatr. Psychiatry. 2002; 17:388-390. [PubMed: 11994895]

37. Pearson RCA, Esiri MM, Hiorns RW, et al. Anatomical correlates of the distribution of the pathological changes in the neocortex in Alzheimer's disease. Proc. Natl. Acad. Sci. USA. 1985; 82:4531-4534. [PubMed: 3859874]

38. Arnold SE, Hyman BT, Flory J, et al. The topographical and neuroanatomical distribution of neurofibrillary tangles and neuritic plaques in the cerebral cortex of patients with Alzheimer's disease. Cereb. Cortex. 1991; 1:103-116. [PubMed: 1822725]

39. Brun A, Englund E. Regional pattern of degeneration in Alzheimer's disease: neuronal loss and histopathologic grading. Histopathology. 1981; 5:549-564. [PubMed: 7286917]

40. Hyman BT, Van Hoesen GW, Damasio AR. Memory-related neural systems in Alzheimer's disease: an anatomic study. Neurology. 1990; 40:1721-1730. [PubMed: 2234428]

41. Mesulam MM. A plasticity-based theory of the pathogenesis of Alzheimer's disease. Ann. N.Y. Acad. Sci. 2000; 924:42-52. [PubMed: 11193801]

42. Gogtay N, Giedd JN, Lusk L, et al. Dynamic mapping of human cortical development during childhood and adolescence. Proc. Natl. Acad. Sci. 2004; 101:8174-8179. [PubMed: 15148381]

43. Yakovlev, PI.; Lecours, AR. The myelogenetic cycles of regional maturation of the brain. In: Minkowski, A., editor. Regional Development of the Brain in Early Life. Oxford: Blackwell Scientific; 1967. p. 3-70.

44. Benes FM, Turtle M, Khan Y, Farol P. Myelination of a key relay zone in the hippocampal formation occurs in the human brain during childhood, adolescence, and adulthood. Arch. Gen. Psychiatry. 1994; 51:477-484. [PubMed: 8192550]

45. Thompson PM, Vidal C, Giedd JN, et al. Mapping adolescent brain change reveals dynamic wave of accelerated gray matter loss in very early-onset schizophrenia. Proc. Natl. Acad. Sci. USA. 2001; 98:11650-11655. [PubMed: 11573002]

46. Cannon TD, Thompson PM, van Erp T, et al. Cortex mapping reveals heteromodal gray matter deficits in monozygotic twins discordant for schizophrenia. Proc. Natl. Acad. Sci. USA. 2002; 99:3228-3233. [PubMed: 11867725]

47. Pantelis C, Velakoulis D, McGorry PD, et al. Neuroanatomical abnormalities before and after onset of psychosis: a cross-sectional and longitudinal MRI comparison. Lancet. 2003; 361:281288. [PubMed: 12559861] 
48. Sun D, Velakoulis D, Yung A, et al. Brain structural change during the development of psychosis: a longitudinal MRI study. 2007 submitted.

49. Bartzokis G, Cummings JL, Sultzer D, et al. White matter structural integrity in healthy aging adults and patients with Alzheimer disease: a magnetic resonance imaging study. Arch. Neurol. 2003; 60:393-398. [PubMed: 12633151]

50. Bartzokis G, Sultzer D, Lu PH, et al. Heterogeneous age-related breakdown of white matter structural integrity: implications for cortical "disconnection" in aging and Alzheimer's disease. Neurobiol. Aging. 2004; 25:843-851. [PubMed: 15212838]

51. Salat DH, Buckner RL, Snyder AZ, et al. Thinning of the cerebral cortex in aging. Cereb. Cortex. 2004; 14:721-730. Epub 2004 Mar 28. [PubMed: 15054051]

52. Ballmaier M, O’Brien JT, Burton EJ, et al. Comparing gray matter loss profiles between dementia with Lewy bodies and Alzheimer's disease using cortical pattern matching: diagnosis and gender effects. Neuroimage. 2004a; 23:325-335. [PubMed: 15325380]

53. Harvey GT, Hughes J, McKeith IG, et al. Magnetic resonance imaging differences between dementia with Lewy bodies and Alzheimer's disease: a pilot study. Psychol. Med. 1999; 29:181187. [PubMed: 10077306]

54. McKeith IG, Dickson DW, Lowe J, et al. Diagnosis and management of dementia with Lewy bodies: third report of the DLB Consortium. Neurology. 2005; 65:1863-1872. Epub 2005 Oct 19. [PubMed: 16237129]

55. Masliah E, DeTeresa RM, Mallory ME, Hansen LA. Changes in pathological findings at autopsy in AIDS cases for the last 15 years. AIDS. 2000; 14:69-74. [PubMed: 10714569]

56. Apostolova LG, Lu P, Rogers S, et al. 3D mapping of language networks in clinical and preclinical Alzheimer's disease. 2007 [submitted].

57. Cummings, JL.; Apostolova, LG.; Akopyan, GG., et al. Structural correlates of apathy in Alzheimer's Disease; Annual Meeting of the American Academy of Neurology; San Diego, CA. 2006.

58. Lu, A.; Leow, AD.; Lee, AD., et al. Growth pattern abnormalities in childhood-onset schizophrenia visualized using tensor-based morphometry. 12th Annual Meeting of the Organization for Human Brain Mapping (OHBM); June 11-15; Florence, Italy. 2006.

59. Thompson PM, Cannon TD, Narr KL, et al. Genetic influences on brain structure. Nat. Neurosci. 2001; 4:1253-1258. [PubMed: 11694885]

60. Gray JR, Thompson PM. Neurobiology of intelligence: science and ethics. Nat. Rev. Neurosci. 2004; 5:471-482. [PubMed: 15152197]

61. McDaniel, MA.; Nguyen, NT. A meta-analysis of the relationship between MRI-assessed brain volume and intelligence. Presented at Proc Int. Soc. Intel. Res.; Nashville, TN. 2002.

62. Ballmaier M, Kumar A, Thompson PM, et al. Localizing gray matter deficits in late onset depression using computational cortical pattern matching methods. Am. J. Psychiatry. 2004; 161:2091-2099. [PubMed: 15514411]

63. Pievani, M.; Testa, C.; Sabattoli, F., et al. Structural correlates of age at onset in Alzheimer's disease: a cortical pattern matching study. 12th Annual Meeting of the Organization for Human Brain Mapping (OHBM); June 11-15; Florence, Italy. 2006.

64. Fox NC, Cousens S, Scahill R, et al. Using serial registered brain magnetic resonance imaging to measure disease progression in Alzheimer disease: power calculations and estimates of sample size to detect treatment effects. Arch. Neurol. 2000; 57:339-344. [PubMed: 10714659]

65. Baron JC, Chetelat G, Desgranges B, et al. In vivo mapping of gray matter loss with voxel-based morphometry in mild Alzheimer's disease. Neuroimage. 2001; 14:298-309. [PubMed: 11467904]

66. Fox NC, Crum WR, Scahill RI, et al. Imaging of onset and progression of Alzheimer's disease with voxel-compression mapping of serial magnetic resonance images. Lancet. 2001; 358:201205. [PubMed: 11476837]

67. Leow, AD.; Huang, SC.; Geng, A., et al. Inverse Consistent Mapping in 3D Deformable Image Registration: its Construction and Statistical Properties. Information Processing in Medical Imaging (IPMI) 2005; Colorado. July 11-15; Glenwood Springs; 2005. 
68. Medina D, Detoledo-Morrell L, Urresta F, et al. White matter changes in mild cognitive impairment and AD: a diffusion tensor imaging study. Neurobiol. Aging. 2006; 27:663-672. [PubMed: 16005548]

69. Fischl B, Dale AM. Measuring the thickness of the human cerebral cortex from magnetic resonance images. Proc. Natl. Acad. Sci. USA. 2000; 97:11050-11055. [PubMed: 10984517]

70. Sowell ER, Peterson BS, Thompson PM, et al. Sex differences in cortical thickness mapped in 176 healthy individuals between 7 and 87 years. 2006 [Epub ahead of print].

71. Thompson PM, Hayashi KM, de Zubicaray G, et al. Mapping hippocampal and ventricular change in Alzheimer's Disease. Neuroimage. 2004; 22:1754-1766. [PubMed: 15275931]

72. De Santi S, de Leon MJ, Rusinek H, et al. Hippocampal formation glucose metabolism and volume losses in MCI and AD. Neurobiol Aging. 2001; 22:529-539. [PubMed: 11445252]

73. Callen DJ, Black SE, Gao F, et al. Beyond the hippocampus: MRI volumetry confirms widespread limbic atrophy in AD. Neurology. 2001; 57:1669-1674. [PubMed: 11706109]

74. Du AT, Schuff N, Amend D, et al. Magnetic resonance imaging of the entorhinal cortex and hippocampus in mild cognitive impairment and Alzheimer's disease. J. Neurol. Neurosurg. Psychiatry. 2001; 71:441-447. [PubMed: 11561025]

75. Soininen HS, Partanen K, Pitkanen A, et al. Volumetric MRI analysis of the amygdala and the hippocampus in subjects with age-associated memory impairment: correlation to visual and verbal memory. Neurology. 1994; 44:1660-1668. [PubMed: 7936293]

76. Jack CR, Petersen RC, Xu YC, et al. Prediction of AD with MRI-based hippocampal volume in mild cognitive impairment. Neurology. 1999; 52:1397-1403. [PubMed: 10227624]

77. Visser PJ, Verhey FRJ, Hofman PAM, et al. Medial temporal lobe atrophy predicts Alzheimer's disease in patients with minor cognitive impairment. J. Neurol. Neurosurg. Psychiatry. 2002; 72:491-497. [PubMed: 11909909]

78. Pennanen C, Kivipelto M, Tuomainen S, et al. Hippocampus and entorhinal cortex in mild cognitive impairment and early AD. Neurobiol. Aging. 2004; 25:303-310. [PubMed: 15123335]

79. Dickerson BC, Salat DH, Bates JF, et al. Medial temporal lobe function and structure in mild cognitive impairment. Ann Neurol. 2004; 56:27-35. [PubMed: 15236399]

80. Killiany RJ, Hyman BT, Gomez-Isla T, et al. MRI measures of entorhinal cortex vs hippocampus in preclinical AD. Neurology. 2002; 58:1188-1196. [PubMed: 11971085]

81. de Leon MJ, Golomb J, George AE, et al. The radiologic prediction of Alzheimer disease: the atrophic hippocampal formation. AJNR Am. J. Neuroradiol. 1993; 14:897-906. [PubMed: 8352162]

82. Visser PJ, Scheltens P, Verhey FRJ, et al. Medial temporal lobe atrophy and memory dysfunction as predictors for dementia in subjects with mild cognitive impairment. J. Neurol. 1999; 246:477485. [PubMed: 10431775]

83. Laakso MP, Lehtovirta M, Partanen K, et al. Hippocampus in AD: a 3-year follow-up MRI study. Biol. Psychiatry. 2000; 47:557-561. [PubMed: 10715362]

84. Killiany RJ, Gomez-Isla T, Moss M, et al. Use of structural magnetic resonance imaging to predict who will get Alzheimer's disease. Ann. Neurol. 2000; 47:430-439. [PubMed: 10762153]

85. Csernansky JG, Wang L, Joshi S, et al. Early DAT is distinguished from aging by high dimensional mapping of the hippocampus. Neurology. 2000; 55:1636-1643. [PubMed: 11113216]

86. Becker JT, Hayashi KM, Seaman JL, et al. Alteration in Hippocampal and Caudate Nucleus Structure in HIV/AIDS Revealed by Three-Dimensional Mapping. 2007 [submitted].

87. Bell-McGinty S, Lopez OL, Meltzer CC, et al. Differential cortical atrophy in subgroups of mild cognitive impairment. Arch. Neurol. 2005; 62:1393-1397. [PubMed: 16157746]

88. Frisoni G, Sabattoli F, Lee AD, et al. In vivo neuropathology of the hippocampal formation in AD: a radial mapping MR-based study. Neuroimage. 2006; 32:104-110. [PubMed: 16631382]

89. Narr KL, Bilder RM, Toga AW, et al. Mapping cortical thickness and gray matter concentration in first episode schizophrenia. Cereb. Cortex. 2005; 15:708-719. [PubMed: 15371291]

90. Gogtay N, Nugent TF, Herman D, et al. Dynamic mapping of human hippocampal development during childhood and adolescence. Hippocampus. 2006; 16:664-672. [PubMed: 16826559] 
91. Thompson PM, Hayashi KM, Simon S, et al. Structural abnormalities in the brains of human subjects who use methamphetamine. J. Neurosci. 2004; 24:6028-6036. [PubMed: 15229250]

92. Lin JJ, Salamon N, Lee AD, et al. 3D pre-operative maps of hippocampal atrophy predict surgical outcomes in temporal lobe epilepsy. Neurology. 2005; 65:1094-1097. [PubMed: 16217065]

93. Bearden CE, Thompson PM, Dalwani M, et al. Cortical gray matter density increases in lithiumtreated patients with bipolar disorder. Biol. Psychiatry. 2007 [In press].

94. Butters MA, Aizenstein HJ, Hayashi KM, et al. Three-dimensional mapping reveals decreased volume of the caudate nucleus in late-life depression. Biol. Psychiatry. 2007 [submitted].

95. Van Hoesen GW, Augustinack JC, Dierking J, et al. The parahippocampal gyrus in Alzheimer's disease. Clinical and preclinical neuroanatomical correlates. Ann. N. Y. Acad. Sci. 2000; 911:254274. [PubMed: 10911879]

96. Roybal, DJ.; Dutton, RA.; Hayashi, KM., et al. Mapping ApoE4 and Gender Effects on Hippocampal Atrophic Rates: A Longitudinal MRI Study of Normal Aging, 2005; Annual Scientific Meeting of the American Geriatric Society (AGS); May 11-15; Orlando, FL. 2005.

97. Ashburner J, Csernansky JG, Davatzikos C, et al. Computer-assisted imaging to assess brain structure in healthy and diseased brains. Lancet. 2003; 2:78-88.

98. Good CD, Johnsrude IS, Ashburner J, et al. A voxel-based morphometric study of ageing in 465 normal adult human brains. Neuroimage. 2001; 14:21-36. [PubMed: 11525331]

99. Ashburner J, Friston KJ. Voxel-based morphometry-the methods. Neuroimage. 2000; 11:805-821. [PubMed: 10860804]

100. Ashburner J, Friston KJ. Why voxel-based morphometry should be used. Neuroimage. 2001; 14:1238-1243. [PubMed: 11707080]

101. Bookstein F. Voxel-based morphometry should not be used with imperfectly registered images. Neuroimage. 2001; 14:1454-1462. [PubMed: 11707101]

102. Davatzikos C, Genc A, Xu D, Resnick SM. Voxel-based morphometry using the RAVENS maps: methods and validation using simulated longitudinal atrophy. Neuroimage. 2001a; 14:13611369. [PubMed: 11707092]

103. Thacker, N. 'Tutorial: A Critical Analysis of VBM,'. 2003. http://www.tina-vision.net/docs/memos/2003-011.pdf, 2003

104. Crum WR, Griffin LD, Hill DL, Hawkes DJ. Zen and the art of medical image registration: correspondence, homology, and quality. Neuroimage. 2003; 20:1425-1437. Review. [PubMed: 14642457]

105. Sowell ER, Thompson PM, Welcome SE, et al. Cortical abnormalities in children and adolescents with attention-deficit hyperactivity disorder. Lancet. 2003; 362:1699-1707. [PubMed: 14643117]

106. Selemon LD, Rajkowska G, Goldman-Rakic PS. Abnormally high neuronal density in the schizophrenic cortex. A morphometric analysis of prefrontal area 9 and occipital area 17. Arch. Gen. Psychiatry. 1995; 52:805-818. discussion 819-20. [PubMed: 7575100]

107. Raz N, et al. Selective aging of the human cerebral cortex observed in vivo: differential vulnerability of the prefrontal gray matter. Cereb. Cortex. 1997; 7:268-282. [PubMed: 9143446]

108. Magnotta VA, et al. Quantitative in vivo measurement of gyrification in the human brain: changes associated with aging. Cereb. Cortex. 1999; 9:151-160. [PubMed: 10220227]

109. Shaw P, Greenstein D, Lerch J, et al. Intellectual ability and cortical development in children and adolescents. Nature. 2006; 440:676-679. [PubMed: 16572172]

110. Vidal CN, Hayashi KM, Geaga JA, et al. Dynamically spreading frontal and cingulate deficits mapped in adolescents with schizophrenia. Arch. Gen. Psychiatry. 2006; 63:25-34. [PubMed: 16389194]

111. Luders, E.; Narr, KL.; Thompson, PM., et al. Gender Effects on Cortical Thickness; 11th Annual Meeting of the Organization for Human Brain Mapping (OHBM); Toronto, Canada. 2005. p. 12-16.

112. Lerch JP, Pruessner JC, Zijdenbos A, et al. Focal decline of cortical thickness in Alzheimer's disease identified by computational neuroanatomy. Cereb Cortex. 2004; 15:995-1001. Epub 2004 Nov 10. [PubMed: 15537673] 
113. Narr KL, Toga AW, Szeszko P, et al. Cortical thinning in cingulate and occipital cortices in first episode schizophrenia. Biol. Psychiatry. 2005; 58:32-40. [PubMed: 15992520]

114. Sowell ER, Thompson PM, Leonard CM, et al. Longitudinal mapping of cortical thickness and brain growth in normal children. J. Neurol. Sci. 2004; 24:8223-8231.

115. Lu LH, Leonard CM, Thompson PM, et al. Normal developmental changes in inferior frontal gray matter are associated with improvement in phonological processing: a longitudinal MRI analysis. Cerebral Cortex. 2006 [June 16 Epub ahead of print.].

116. Sowell ER, Thompson PM, Mattson SN, et al. Regional brain shape abnormalities persist into adolescence after heavy prenatal alcohol exposure. Cereb. Cortex. 2002a; 12:856-865. [PubMed: 12122034]

117. Gogtay N, Ordonez A, Herman DH, et al. Dynamic mapping of cortical brain development in pediatric bipolar illness. J. Child Psychol. Psychiatry. 2007 [In press].

118. Bearden, CE.; Dutton, RA.; van Erp, TGM., et al. Abnormal cortical thickness and cortical asymmetry mapped in children with 22q11.2 Microdeletions; 12th Annual Meeting of the Organization for Human Brain Mapping (OHBM); June 11-15; Florence, Italy. 2006.

119. Lin JJ, Salamon N, Lee AD, et al. Reduced Cortical Thickness \& Complexity Mapped in Mesial Temporal Lobe Epilepsy with Hippocampal Sclerosis. Cerebral Cortex. 2006 [In press].

120. Cannon TD, Hennah W, van Erp TGM, et al. DISC1/TRAX haplotypes associate with schizophrenia, reduced prefrontal gray matter, and impaired short- and long-term memory. Arch. Gen. Psychiatry. 2005; 62:1205-1213. [PubMed: 16275808]

121. Zeineh MM, Engel SA, Thompson PM, Bookheimer S. Dynamics of the hippocampus during encoding and retrieval of face-name pairs. Science. 2003; 299:577-580. [PubMed: 12543980]

122. Le Bihan D, Breton E, Lallemand D, et al. MR imaging of intravoxel incoherent motions: application to diffusion and perfusion in neurologic disorders. Radiology. 1986; 161:401-407. [PubMed: 3763909]

123. Moseley ME, Cohen Y, Kucharczyk J, et al. Diffusion-weighted MR imaging of anisotropic water diffusion in cat central nervous system. Radiology. 1990; 176:439-445. [PubMed: 2367658]

124. Basser PJ, Mattiello J, LeBihan D. Estimation of the effective self-diffusion tensor from the NMR spin echo. J. Magn. Reson. B. 1994; 103:247-254. [PubMed: 8019776]

125. Kantarci K, Jack CR Jr, Xu YC, et al. Regional metabolic patterns in mild cognitive impairment and Alzheimer's disease: A 1H MRS study. Neurology. 2000; 55:210-217. [PubMed: 10908893]

126. Kantarci K, Jack CR Jr, Xu YC, et al. Mild cognitive impairment and Alzheimer disease: regional diffusivity of water. Radiology. 2001; 219:101-107. [PubMed: 11274543]

127. Sandson TA, Felician O, Edelman RR, Warach S. Diffusion-weighted magnetic resonance imaging in Alzheimer's disease. Dement. Geriatr. Cogn. Disord. 1999; 10:166-171. [PubMed: 10026392]

128. Schott JM, Price SL, Frost C, et al. Measuring atrophy in Alzheimer disease: a serial MRI study over 6 and 12 months. Neurology. 2005; 65:119-124. [PubMed: 16009896]

129. Thompson PM, Giedd JN, Woods RP, et al. Growth patterns in the developing brain detected by using continuum-mechanical tensor maps. Nature. 2000; 404:190-193. [PubMed: 10724172]

130. Janke AL, Zubicaray GD, Rose SE, et al. 4D deformation modeling of cortical disease progression in Alzheimer's dementia. Magn Reson Med. 2001; 46:661-666. [PubMed: 11590641]

131. Studholme, C.; Cardenas, V.; Schuff, N., et al. Detecting Spatially Consistent Structural Differences in Alzheimer's and Fronto Temporal Dementia Using Deformation Morphometry; Conference Series on Medical Imaging Computing and Computer-Assisted Intervention (MICCAI) 2001; 2001. p. 41-48.

132. Chiang, MC.; Leow, AD.; Dutton, RA., et al. Fluid registration of diffusion tensor imaging using information theory. In: Gee, JC.; Thompson, PM., editors. Submitted to IEEE Transactions on Medical Imaging, Special Issue on Computational Neuroanatomy. 2006. to appear March 2007 [submitted, June 29, 2006]

133. Leow AD, Soares JC, Hayashi KM, et al. Asymmetrical effects of lithium on brain structure mapped in healthy individuals. 2006 [submitted]. 
134. Thompson PM, Toga AW. Cortical diseases and cortical localization [Review Article]. Nature Encyclopedia of the Life Sciences (ELS), 2003. 2003

135. Leow, AD.; Lee, AD.; Chiang, MC., et al. Analysis of Regional Brain Atrophy in a Single Case of Semantic Dementia Using Serial MRI with Inverse-Consistent Non-Rigid Registration; 11th Annual Meeting of the Organization for Human Brain Mapping (OHBM); June 12-16; Toronto, Canada. 2005.

136. Lee, AD.; Leow, AD.; Lu, A., et al. Tensor-Based Morphometry Reveals 3D Profile of Altered Brain Structure in Fragile X Syndrome; 61st Annual Scientific Convention of the Society of Biological Psychiatry (SOBP); May 18-20; Toronto, Ontario, Canada. 2006.

137. Chiang, MC.; Reiss, AL.; Dutton, RA., et al. 3D Pattern of Brain Volume Reduction in Williams Syndrome Visualized using Tensor-Based Morphometry; 12th Annual Meeting of the Organization for Human Brain Mapping (OHBM); June 11-15; Florence, Italy. 2006.

138. Foland, LC.; Altshuler, LL.; Leow, AD., et al. A Tensor-Based Morphometric Study of Bipolar Disorder; 12th Annual Meeting of the Organization for Human Brain Mapping (OHBM); June 11-15; Florence, Italy. 2006.

139. Lepore, N.; Chou, YY.; Brun, CA., et al. Genetic Influences on Brain Structure and Fiber Architecture Mapped Using Diffusion Tensor Imaging and Tensor-Based Morphometry in Twins; 12th Annual Meeting of the Organization for Human Brain Mapping (OHBM); June 1115; Florence, Italy. 2006.

140. Jack CR Jr, Petersen RC, Xu Y, et al. Rates of hippocampal atrophy correlate with change in clinical status in aging and AD. Neurology. 2000; 55:484-489. [PubMed: 10953178]

141. Bernstein, MA.; Lin, C.; Borowski, BJ., et al. Alzheimer's Disease Neuroimaging Initiative (ADNI): The MR Imaging Protocol. Presented at State-of-the-Art Cardiovascular and Neuro MRI, a Joint Workshop of the ISMRM and CSR; September; Beijing, China. 2005.

142. Archer HA, Edison P, Brooks DJ, et al. Amyloid load and cerebral atrophy in Alzheimer's disease: An (11)C-PIB positron emission tomography study. Ann Neurol. 2006; 60:145-147. [Epub ahead of print]. [PubMed: 16802294]

143. Kochunov PK, et al. Relationship among neuroimaging markers of merebral atrophy during normal aging. Human Brain Mapping. 2007 Feb 8. [Epub ahead of print].

144. Jack CR Jr, Petersen RC, Xu YC, et al. Medial temporal atrophy on MRI in normal aging and very mild Alzheimer's disease. Neurology. 1997; 49:786-794. [PubMed: 9305341]

145. Xu Y, et al. Usefulness of MRI measures of entorhinal cortex versus hippocampus in AD. Neurology. 2000; 54(9):1760-1767. [PubMed: 10802781]

146. Chetelat G, Desgranges B, De La Sayette V, et al. Mapping gray matter loss with voxel-based morphometry in mild cognitive impairment. Neuroreport. 2002; 13:1939-1943. [PubMed: 12395096]

147. Kochunov P, et al. Age-related morphology trends of cortical sulci. Human Brain Mapping. 2005; 26(3):210-220. [PubMed: 16161162]

148. Burggren AC, et al. Reduced cortical thickness in hippocampal subregions in people at genetic risk for Alzheimer's disease. 2007 [submitted].

149. Rose SE, et al. Diffusion indices on magnetic resonance imaging and neuropsychological performance in amnestic mild cognitive impairment. J. Neurol. Neurosurg. Psychiatry. 2006; 77(10):1122-1128. [PubMed: 16754694] 


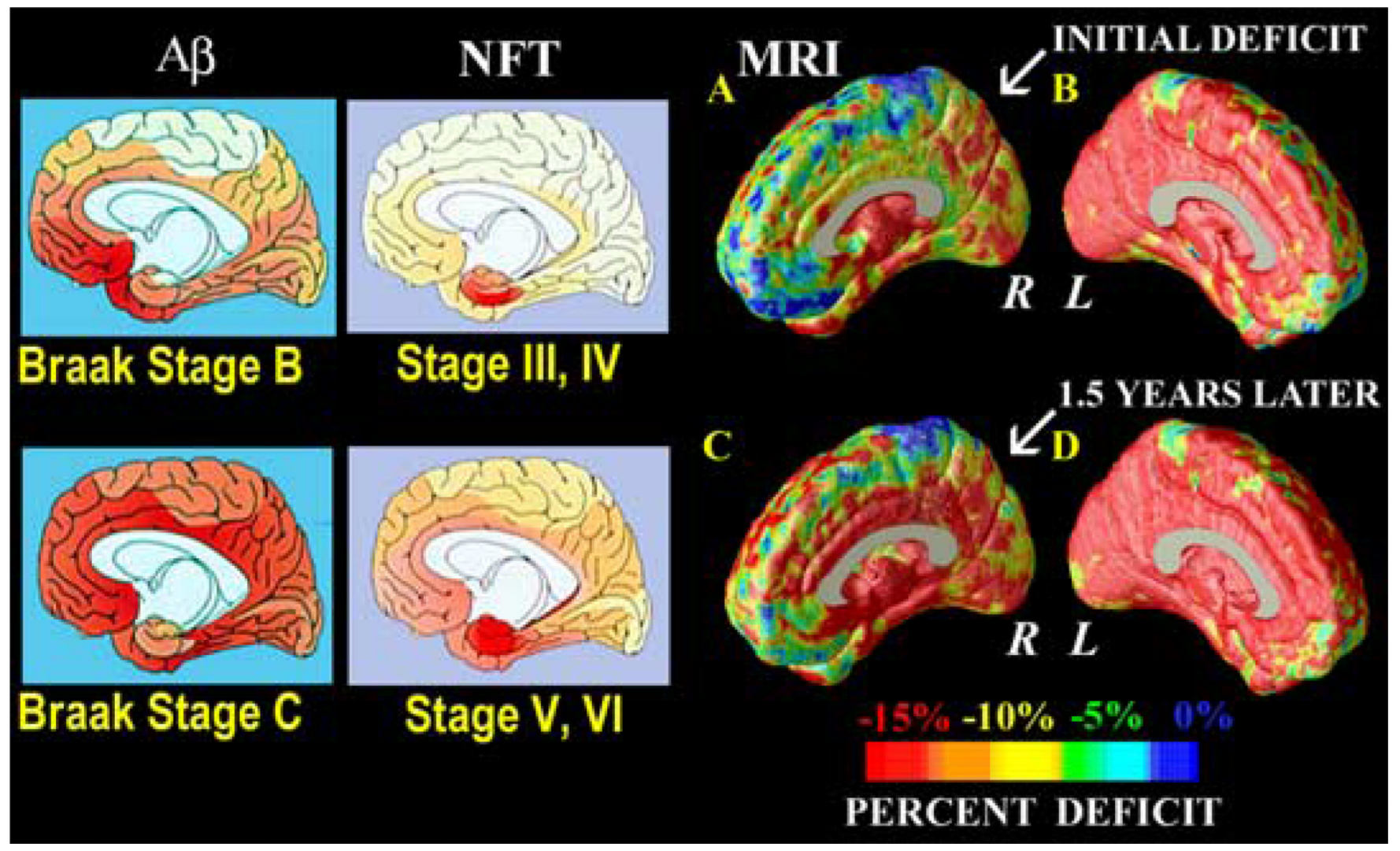

FIGURE 1.

Gray matter deficits spread through the limbic system in moderate AD. Cortical atrophy occurring during the progression of $\mathrm{AD}$ is detected by comparing average profiles of gray matter between 12 AD patients (age: $68.4 \pm 1.9$ years) and 14 elderly matched controls (age: $71.4 \pm 0.9$ years). Average maps of gray matter density in patients and controls are subtracted at their first scan (when mean Mini-Mental State Exam (MMSE) score was 18 for the patients; [A] and [B]) and at their follow-up scan 1.5 years later (mean MMSE $=13$; $[\mathbf{C}]$ and $[\mathbf{D}]$ ). Colors show the average percentage loss of gray matter relative to the control average. Profound loss engulfs the left medial wall (>15\%; $[\mathbf{B}]$ and $[\mathbf{D}])$. On the right, however, the deficits in temporoparietal and entorhinal territory (A) spread forward into the cingulate gyrus 1.5 years later $(\mathbf{C})$, after a 5-point drop in average MMSE. Limbic and frontal zones clearly show different degrees of impairment $(\mathbf{C})$. The corpus callosum is indicated in white; maps of gray matter change are not defined here, as it is a white matter commissure. MRI-based changes, in living patients, agree strongly with the spatial progression of $\beta$-amyloid (A $\beta$ ) and NFT pathology observed post mortem (Braak Stages B, $C$ and III to VI; left four panels adapted from Braak and Braak, 1997 ${ }^{30}$ ). The deficit sequence also matches the trajectory of NFT distribution observed post mortem, in patients with increasing dementia severity at death. ${ }^{30}$ Consistent with the deficit maps observed here, NFT accumulation is minimal in sensory and motor cortices, but occurs preferentially in entorhinal pyramidal cells, the limbic periallocortex (layers II/IV), the hippocampus/ amygdala and subiculum, the basal forebrain cholinergic systems, and subsequently in temporoparietal and frontal association cortices (layers III/V). ${ }^{37,38}$ Cortical layers III and V selectively lose large pyramidal neurons in association areas. ${ }^{39,40}$ This figure appears in color online. 


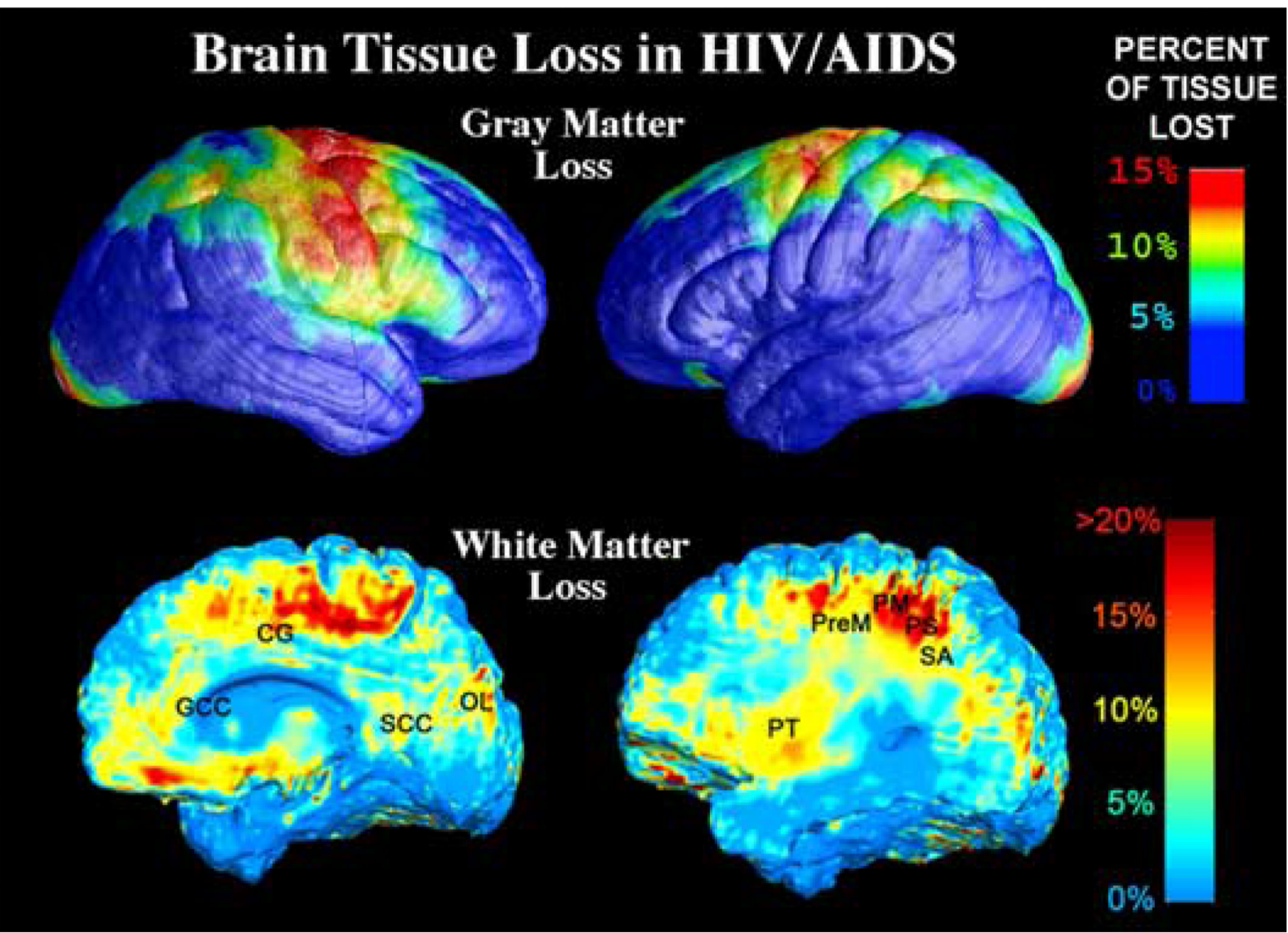

FIGURE 2.

Visualizing brain tissue loss in HIV/AIDS. (Top row): In an MRI study of cortical thickness in 27 HIV/AIDS patients and 14 healthy controls, the primary sensory, motor, and premotor cortices were $15 \%$ thinner, and prefrontal and parietal tissue loss correlated with cognitive and motor deficits. Thinner frontopolar and language cortex also correlated with immune system deterioration measured via blood levels of $\mathrm{CD}^{+}{ }^{+} \mathrm{T}$-lymphocytes. (Bottom row): When the same subjects were studied using TBM, ${ }^{9}$ the pattern of white matter loss was in remarkable agreement with the cortical maps. The white matter volume was reduced in premotor areas where the cortex was significantly thinner, suggesting that cortical degeneration may be accompanied by degeneration in the underlying white matter pathways. Taken together, these and other studies support the notion that brain degeneration is present even in apparently healthy HIV-positive people on powerful drug regimens (HAART; highly active antiretroviral therapy). (Data in the top row are from Thompson et al., 20057 data in the bottom row are from Chiang et al., 2006 ${ }^{9}$ ). 


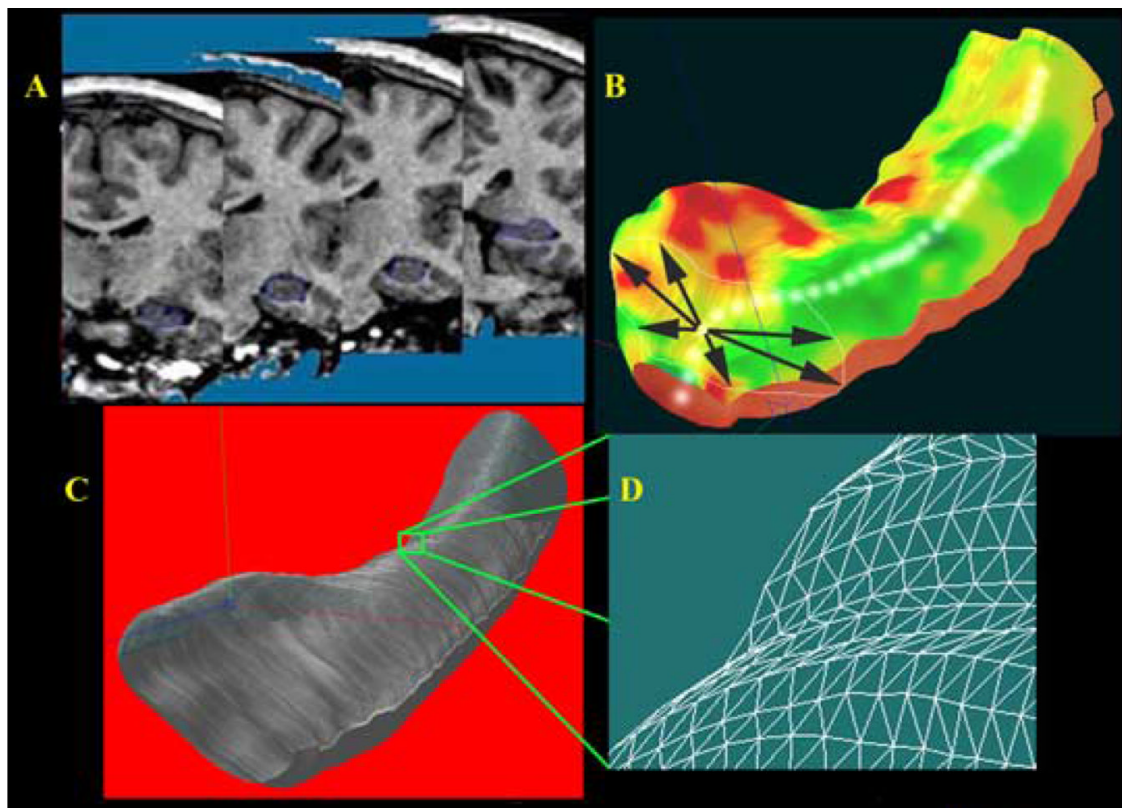

\section{ELDERLY CONTROLS}
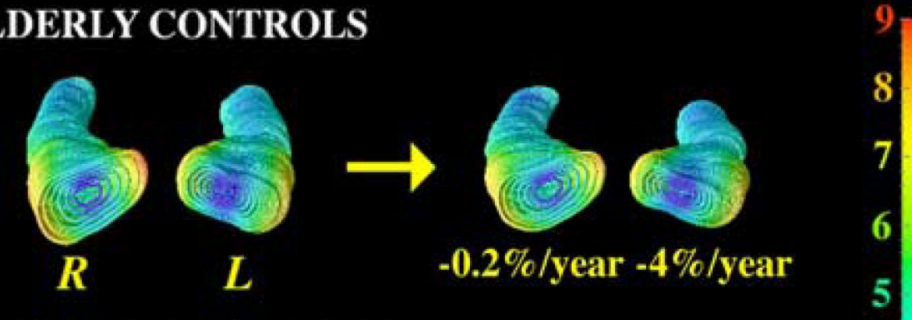

ALZHEIMER'S DISEASE
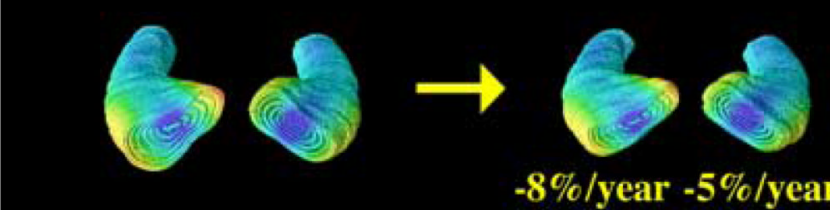

4

3<smiles>C1CCCCC1</smiles>

\section{DIFFERENCE}
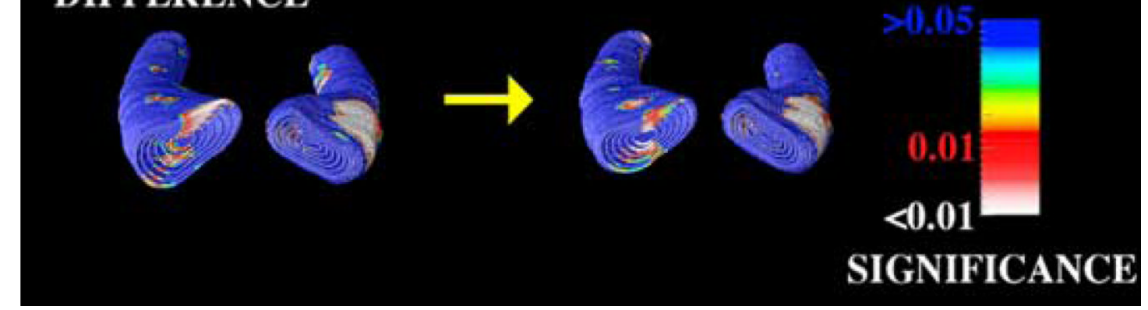

Radial Size

(mm)

FIGURE 3 .

Mapping hippocampal atrophy. The 3D profile of hippocampal atrophy in disease can be mapped using surface-based modeling methods. ${ }^{71}$ The hippocampus is traced (A) either by hand or automatically, in serial coronal sections. After converting the traces to parametric surface mesh format $(\mathbf{C})$ and (D), a medial core (i.e., a curve threading down the center of the hippocampus) is computed for each hippocampus. The distances from the medial core to each surface point are estimated and used to generate first individual and later average group distance maps. Here, group distance maps for elderly controls and patients with moderate Alzheimer's disease are compared at baseline (left column), and after an approximately 2year follow-up interval (right column). The significance maps show regions with significant 
atrophy at each time point (white colors). Similar maps can be made to plot regions where there is evidence for progressive atrophy over time or changes that link with cognitive test performance or clinical outcomes. ${ }^{92}$ 

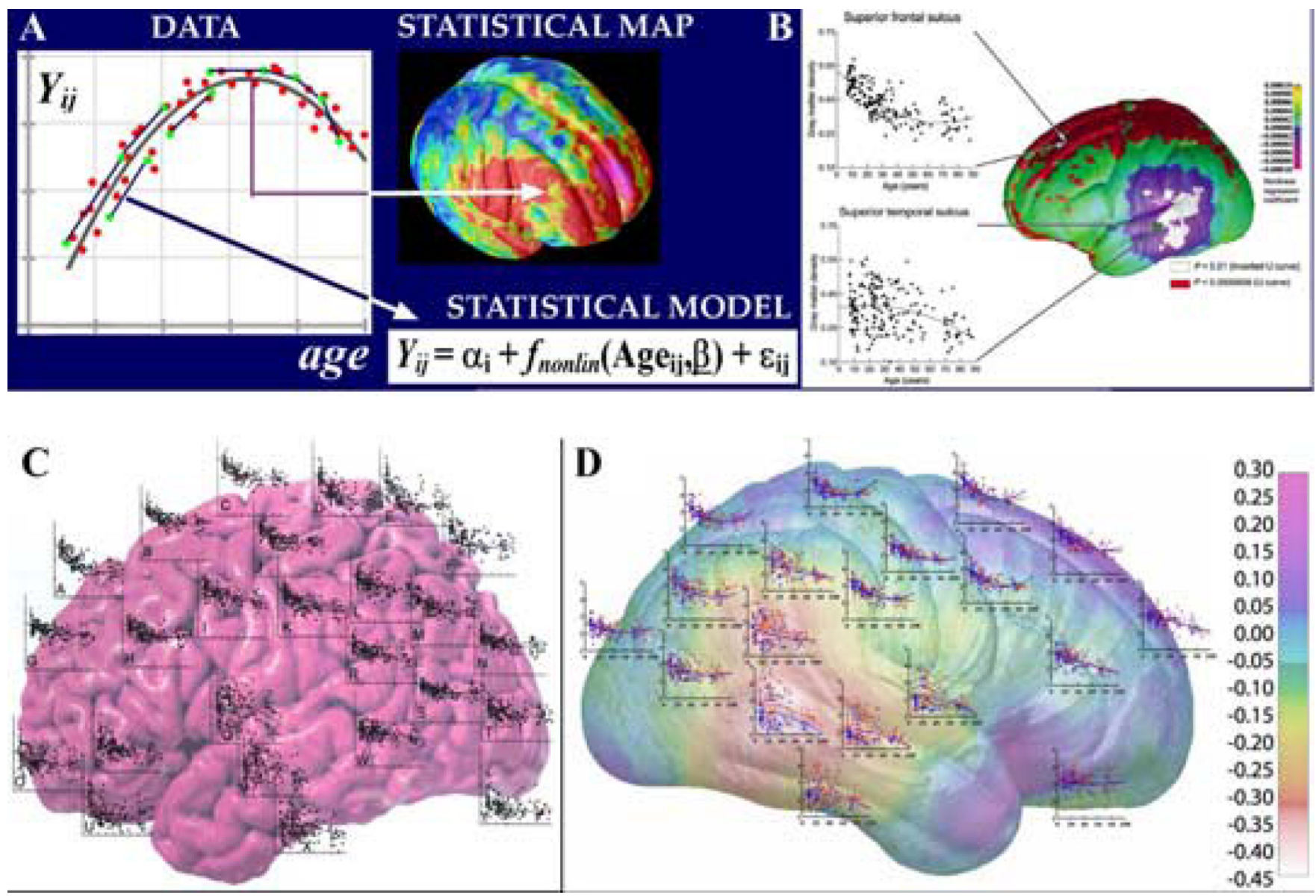

FIGURE 4.

Mapping cortical changes. Several recent studies have examined the effects of age on the thickness of the cortex, measured in MRI scans of the brain. 5,7,70,109 This figure shows a general approach we developed to map cortical gray matter changes over time; it has also been used to produce time-lapse animations of the trajectory of cortical thinning with age, and to map the progression of diseases, such as childhood onset schizophrenia and Alzheimer's disease. ${ }^{28,45,110}$ (A) First, measures $\left(Y_{i j}\right)$ of gray matter thickness are obtained longitudinally (green dots) or once only (red dots) in a group of subjects at different ages. Fitting of statistical models to these data (Statistical Model, lower right) produces estimates of parameters that can be plotted onto the cortex, using a color code. These parameters can include age at peak (see arrow at peak of the curve), significance values, or estimated statistical parameters, such as rates of change, and effects of drug treatment or risk genes. (B) and (C): We estimated the trajectory of gray matter loss over the human life span in a cohort of 176 normal subjects. ${ }^{5}$ After cortical pattern matching was used to associate data from corresponding cortical regions, we developed software to fit a general nonlinear statistical model to the gray matter data from the population. This revealed significant nonlinear (quadratic) effects of time on brain structure. To show that it is feasible to pick up very small systematic differences using this technique, Sowell et al. (2006) found a sex difference in the trajectory of cortical thinning (D). There was an absolute excess in cortical thickness in women relative to men, of around $0.3 \mathrm{~mm}$, mainly in the perisylvian language areas (even without brain size adjustments). Our other cortical thickness studies provide evidence for this sex difference, ${ }^{111}$ which is important to consider if cortical thickness is 
used to gauge degenerative brain changes. For full details of the approach, see Refs. 12,70. This figure appears in color online. 


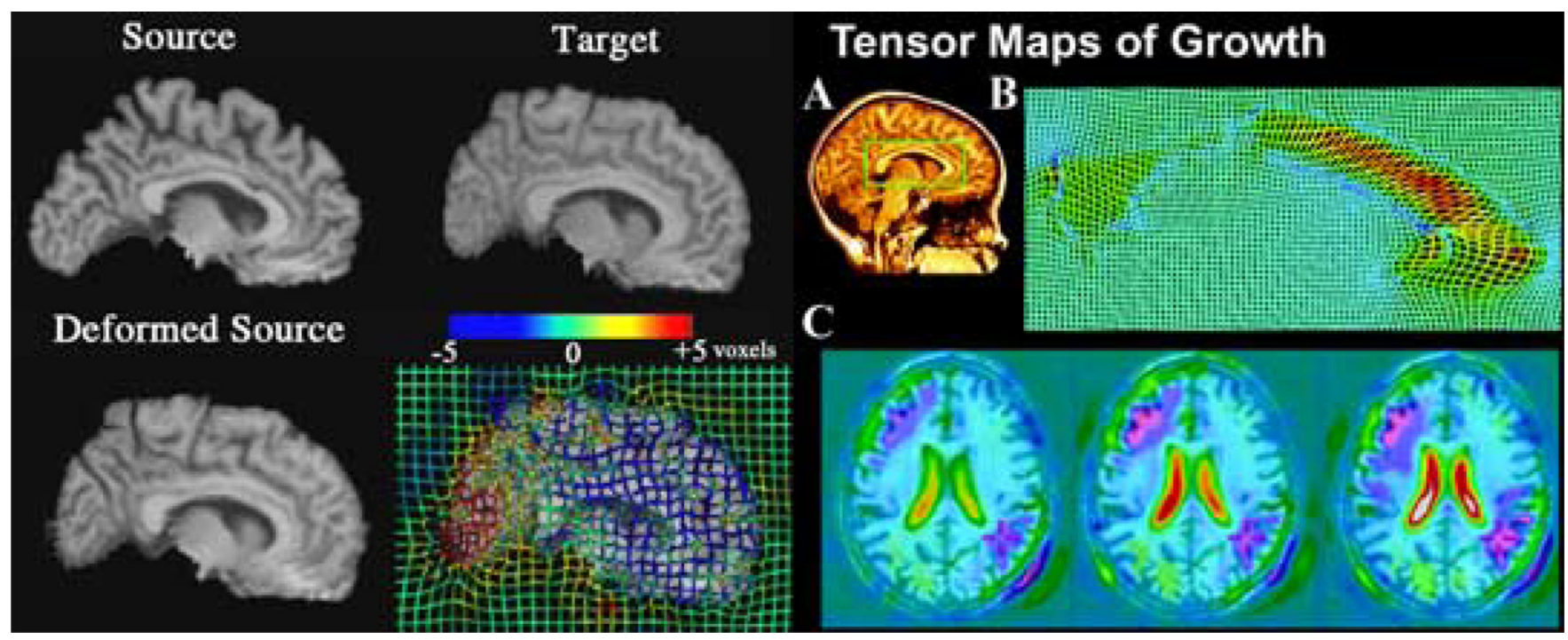

FIGURE 5.

TBM maps rates of tissue gain or loss. Tensor-based morphometry, or TBM, can automatically map brain changes in large groups of subjects. Here, an example MRI image (top left) is aligned to a target image, and the shape of gyri, corpus callosum, and ventricles are well matched (data from Ref. 9). To better visualize the applied 3D deformation field, a colored grid is superimposed on the registered image — red and blue colors represent deformation orthogonal to the midsagittal plane (out of the page). (A) and (B): In Thompson et al. (2000), we used TBM to map growth rates (B) in the corpus callosum (A) of a young girl scanned at 3 years of age and again at 6 years-the anterior corpus callosum grows the fastest at this age ( $20 \%$ local volume gain per year, most likely due to progressive myelination). Rates of tissue expansion are inferred from the derivatives of the warping field that aligns baseline to follow-up scans. (C): Progressive gray and white matter atrophy (purple colors) and ventricular expansion (red and white colors) are mapped in a patient with posterior cortical atrophy scanned 1, 1.5, and 2 years after diagnosis. After registering growth rate maps to a common neuroanatomical template, group differences in rates of brain change can be tested statistically (see Refs. 25 and 58, for examples of studies comparing groups cross-sectionally and longitudinally). This figure appears in color online. 